\title{
Spin $M 1$ excitations in deformed nuclei from self-consistent Hartree-Fock plus random-phase approximation
}

\author{
P. Sarriguren, E. Moya de Guerra, and R. Nojarov* \\ Instituto de Estructura de la Materia, Consejo Superior de Investigaciones Científicas, Serrano 119, E-28006 Madrid, Spain \\ (Received 25 October 1995; revised manuscript received 23 April 1996)
}

\begin{abstract}
We present a method to study spin magnetic dipole excitations in deformed nuclei within the quasiparticle random phase approximation based on self-consistent Hartree-Fock mean fields and residual interactions derived from the same effective two-body force. We perform a comprehensive study covering different Skyrme forces and various mass regions, and discussing the role of the mean field and of the residual interaction. An overall agreement with experimental data is obtained with the SG2 force. We study the systematics and the deformation dependence of the spin $M 1$ strength distributions of $K^{\pi}=1^{+}$excitations. It is found for the first time that the summed spin $M 1$ strength obeys a quadratic dependence on deformation in the two isotope chains studied, ${ }^{142,146,148,150} \mathrm{Nd}$ and ${ }^{144,148,150,152,154} \mathrm{Sm}$. [S0556-2813(96)03908-8]

PACS number(s): 21.60.Jz, 21.10.Re, 27.60.+j, 27.70.+q
\end{abstract}

\section{INTRODUCTION}

The discovery of magnetic dipole excitations in eveneven deformed nuclei by Bohle et al. in 1984 [1] initiated a new line of investigations in nuclear structure that is still actively pursued. The discovery was done by high-resolution inelastic electron scattering from rare-earth nuclei. The investigations were complemented soon afterwards with other experiments involving different probes and mass regions. Numerous electron scattering experiments have been performed since then to study the systematics and fragmentation of this new low-lying mode (see, for example, the review articles in Ref. [2]). Nuclear resonance fluorescence (NRF) has been also very useful because it constitutes a highly selective and sensitive probe to investigate the low-lying dipole excitations, showing an important fragmentation of this mode [3]. Inelastic proton scattering at forward angles was performed [4] to study the spin contributions. It confirmed the predominantly orbital nature of the low-lying $1^{+}$states. The combined analysis of all these high-resolution inelastic scattering experiments established the existence of this mode as a general phenomenon in deformed even-even nuclei. The basic features of the low-lying excitations are nowadays well known (see, for example [5,6], and the review articles [2,7]).

The $\left(p, p^{\prime}\right)$ measurements were first performed to study the small spin contributions at low energy, confirming the orbital nature of the low-lying states. More recently, an experimental study of the spin magnetic dipole strengths in medium heavy and heavy deformed nuclei has been started using both unpolarized and polarized protons. Inelastic scattering experiments [8] of medium-energy protons at extreme forward angle indicate the existence of considerable spin strength (about $11 \mu_{N}^{2}$ ) between excitation energies ranging from 5 to $10 \mathrm{MeV}$ in several rare-earth nuclei. The distribu-

*Permanent address: Institute for Nuclear Research and Nuclear Energy, Bulgarian Academy of Sciences, BG-1784, Sofia, Bulgaria. tion of the strength in this energy range exhibits a doublehumped structure in most nuclei. These experiments represent indeed a significant advantage over the electron scattering ones since the latter may not be sensitive enough to the expected excitations in this energy region because of background problems and interference between orbital and spin contributions.

The $M 1$ giant resonance in the spherical nucleus ${ }^{208} \mathrm{~Pb}$ was already known from $\left(e, e^{\prime}\right)$ and $\left(\gamma, \gamma^{\prime}\right)$ reactions. The shell model predicts strong isovector $1^{+}$excitations in nuclei where the $j=l \pm 1 / 2$ spin-orbit partners are located at opposite sides of the Fermi surface. The $M 1$ resonance in ${ }^{208} \mathrm{~Pb}$ is spread around 6-8 MeV [9] and built up essentially by spinflip excitations. The theoretical calculations of Cha et al. [10] were able to describe the quenching and the position and spreading width of the strength distributions by accounting for two-particle-two-hole (2p-2h) correlations, meson exchange currents, and isobar-hole excitations. If the data on ${ }^{208} \mathrm{~Pb}$ are compared with the inelastic proton scattering data on the $M 1$ giant resonance in deformed nuclei, as done in Ref. [7], one can see that the deformed nuclei show a much stronger fragmentation of strength over a broader energy range in comparison with the spherical case. This is due to the higher density of underlying quasiparticle states in the deformed nuclei.

The experimental spin $M 1$ strength in deformed eveneven nuclei is extracted from inelastic $\left(p, p^{\prime}\right)$ experiments at forward angles after subtracting a huge background, whose shape is derived by extrapolation of the low-energy tail of the giant electric dipole resonance. More recently [11,12], experiments with polarized protons have been done in order to eliminate this huge background. In this case, side effects like the Coulomb excitation of the electric giant dipole resonance do not contribute to the spin-flip probability $S_{N N}$. Therefore, it is possible to deduce the $M 1$ strength in a way less dependent on the details of the background in the cross section. But the strong background of quasielastic scattering is present even in the case of polarized protons. 
The analysis of these measurements is still in progress. Preliminary data on ${ }^{154} \mathrm{Sm}[7]$ indicate a reduction of the strength in the first peak as compared to the previous unpolarized proton measurements. This reduction is more in line with our previous microscopic results [13,14] obtained within the quasiparticle random phase approximation (QRPA). These data indicate also the existence of some strength between 10 and $12 \mathrm{MeV}$, not seen previously. It should be mentioned that the extraction of the spin $M 1$ strength from the experimental data has been done assuming a neutron $h_{11 / 2}-h_{9 / 2}$ particle-hole configuration. Hence, the extracted empirical $M 1$ strengths could change when including other possible configurations.

The random phase approximation is widely recognized to be a suitable approach for nuclear structure studies, providing a successful formalism for the description of various nuclear excitations such as giant multipole resonances or low-lying particle-hole excitations. Different theoretical frameworks are used in microscopic RPA calculations depending on the procedure adopted in selecting the mean-field basis and the residual interactions. A common procedure is to take the single-particle energies from experiment and the wave functions from harmonic oscillator or Woods-Saxon potentials, and to adjust phenomenologically the residual forces. Conceptually, it is, however, more appealing to follow a self-consistent procedure and to derive the mean field and the residual interaction from the same effective nucleonnucleon force.

Within the self-consistent approach (Hartree-Fock + RPA), the Skyrme forces are among the most commonly used effective interactions. Although much work has been done in this direction, we would like to mention here only two of the pioneering studies: the work of Vautherin and Brink [15], where the first Hartree-Fock (HF) calculations with Skyrme forces were performed, and the work of Bertsch and Tsai [16], where numerical methods for RPA with Skyrme forces were developed. We apply here for the first time this theoretical framework to the description of $M 1$ excitations in deformed nuclei. Most of the Skyrme forces have been adjusted to nuclear matter and ground-state properties only. Therefore, these forces, and particularly in their original versions, may not be the most appropriate effective forces to describe spin $M 1$ excitations. For this reason we study here several Skyrme forces including the SG2 force, which is known to have good spin properties [17].

The purpose of this work is to study the spin $M 1$ strength distributions in even-even deformed nuclei within a selfconsistent $\mathrm{HF}+\mathrm{RPA}$ formalism with Skyrme forces. Our theoretical approach follows closely the QRPA used in previous works $[13,14,18-20]$, except that here it is based on self-consistent mean fields and spin-spin residual interactions derived from the same effective two-body force. In the past, QRPA calculations have been done by several groups [13,14,18-24] using phenomenological deformed potentials and different residual interactions. In particular, in Refs. $[13,14,20]$ we used deformed Woods-Saxon potentials and separable residual interactions that were relatively successful in describing the phenomenology of $M 1$ excitations. Nevertheless, we got systematically less strength than observed in the first peak of the spin strength distribution. The representation of the effective nucleon-nucleon interaction as a mean field plus residual interactions indicates obviously that the residual interactions are strongly correlated with the choice of the mean field. This correlation is explicitly taken into account here. It is important to note that with the procedure used in this work there are basically no free parameters, apart from the pairing gaps.

Experimental and theoretical studies of spin $M 1$ strength distributions have been so far restricted to a few well deformed nuclei $(\beta \sim 0.3)$ from the rare-earth and actinide regions. This is in contrast to the case of the low-lying, predominantly orbital, $M 1$ excitations that have been extensively investigated and whose systematics is at present well established (see, for instance, [2], and references therein). A very interesting feature that emerged from these investigations is the quadratic dependence on deformation of the summed low-lying $M 1$ strengths [5,6].

In this paper we carry out a more extensive study of spin M1 strength distributions in the above-mentioned mass regions with the aim of establishing their systematics. In particular, it will be interesting to check whether the observed characteristic two-humped structure is still present in transitional nuclei with small deformations. It will also be interesting to see whether the spin $M 1$ strength may depend on global properties such as deformation or mass number. To study the deformation dependence we consider two chains of isotopes in the rare-earth region, ${ }^{144,148,150,152,154} \mathrm{Sm}$ and $142,146,148,150 \mathrm{Nd}$, whose shapes vary from spherical to well deformed. These chains have been already investigated experimentally $[5,6]$ and theoretically [14,25-27] by exploring the deformation dependence of the low-lying $M 1$ strength, a feature that is well accounted for by the present calculations, as shown in Sec. VII.

The paper is organized as follows. In the next section we describe briefly the deformed HF+RPA formalism used in this work, as well as the various Skyrme forces considered. In Secs. III and IV we study the role of the mean field and residual interactions by comparing the results from different Skyrme forces. In Sec. V we compare our results with the available experimental data on the spin $M 1$ strength distributions. In Sec. VI we show results for 24 nuclei and discuss their common and distinguishing features. Finally, in Sec. VII we present a more detailed study of the Nd- and Smisotope chains, where we discuss the deformation dependence of the spin $M 1$ strength distributions as well as of the summed strengths. The main conclusions are summarized in Sec. VIII.

\section{SELF-CONSISTENT HF + RPA FOR DEFORMED NUCLEI}

As mentioned in the Introduction, the RPA formalism can be based on different assumptions for the mean field and the residual interactions. The mean field was taken in previous works $[13,14,19,20]$ from a deformed Woods-Saxon potential and the coupling constants of the residual spin-spin force were derived from Brueckner-Hartree-Fock nuclear matter calculations. In this paper we adopt a self-consistent approach using the same effective interaction to obtain both the self-consistent mean field (with its corresponding singleparticle energies and wave functions) and the residual inter- 
TABLE I. Parameters of the Skyrme forces (1) considered in this work: $t_{0}\left[\mathrm{MeV} \mathrm{fm}^{3}\right], t_{1}\left[\mathrm{MeV} \mathrm{fm}^{5}\right]$, $t_{2}\left[\mathrm{MeV} \mathrm{fm}{ }^{5}\right], t_{3}\left[\mathrm{MeV} \mathrm{fm}^{6}\right], W\left[\mathrm{MeV} \mathrm{fm}{ }^{5}\right], x_{0}, x_{1}, x_{2}, x_{3}$, and $\alpha$.

\begin{tabular}{lcccccccccc}
\hline \hline & $t_{0}$ & $t_{1}$ & $t_{2}$ & $t_{3}$ & $W$ & $x_{0}$ & $x_{1}$ & $x_{2}$ & $x_{3}$ & $1 / \alpha$ \\
\hline SG2 [17] & -2645.0 & 340.0 & -41.9 & 15595.0 & 105.0 & 0.09 & -0.0588 & 1.425 & 0.06044 & 6.0 \\
SG1 [17] & -1603.0 & 515.9 & 84.5 & 8000.0 & 115.0 & -0.02 & -0.5 & -1.731 & 0.1381 & 3.0 \\
Sk3 [31] & -1128.75 & 395.0 & -95.0 & 14000.0 & 120.0 & 0.45 & 0.0 & 0.0 & 1.0 & 1.0 \\
G $_{\sigma}[36]$ & -1800.16 & 336.23 & -85.74 & 11113.5 & 121.86 & -0.4862 & 0.0 & 0.0 & -1.0295 & 3.333 \\
Ska [33] & -1602.78 & 570.88 & -67.7 & 8000.0 & 125.0 & -0.02 & 0.0 & 0.0 & -0.286 & 3.0 \\
Skm* [35] & -2645.0 & 410.0 & -135.0 & 15595.0 & 130.0 & 0.09 & 0.0 & 0.0 & 0.0 & 6.0 \\
\hline \hline
\end{tabular}

actions. This approach is described succinctly in the next subsections.

\section{A. Deformed Hartree-Fock with Skyrme forces}

It is well known that the density-dependent HF approximation gives a very good description of ground-state properties for both spherical and deformed nuclei [28] and it is at present the most reliable mean-field description. The main features of the deformed Hartree-Fock calculations with Skyrme forces are described in this subsection.

Our deformed Hartree-Fock calculations are based on the formalism developed in Ref. [15]. We use the McMaster code that follows the method described in Ref. [29]. Pairing correlations are included in the BCS approximation with fixed gap parameters.

The basis space consists of 11 harmonic oscillator major shells. We work with the full HF bases, i.e., with the whole sets of single-particle wave functions and energies for each nucleus as they come out from the calculations (we do not shift any single-particle energy level).

There are remarkable differences with respect to our previous treatment of the mean field in terms of Woods-Saxon potentials. Among them we can mention the drastic reduction in the number of input parameters: The quadrupole deformation of the ground state $\beta$ is, for instance, determined consistently and no explicit parameter is needed to fit the quadrupole moment. We can also mention the effect of a spin-spin interaction on the self-consistent mean field through the spin exchange operators of the Skyrme force, which is absent in the Woods-Saxon potential. In the present work, once the Skyrme interaction is chosen, no free parameters are left apart from the pairing gaps. In order to reduce the number of parameters, we have used in this work the same pairing gaps for all the rare-earth nuclei considered $\left(\Delta_{p}=\Delta_{n}=1 \mathrm{MeV}\right)$ and the values $\Delta_{p}=\Delta_{n}=0.7 \mathrm{MeV}$ for the actinides, unless otherwise specified.

Because of the success of HF calculations with Skyrme interactions, attempts were made to generalize the force and to apply it also to excited states, covering in this way more features of nuclear structure. Different generalizations [17,30-37] of the original Skyrme forces have been proposed for this purpose, as well as to avoid spin instabilities associated with the zero-range three-body interaction [38]. Some of the generalized forces [32,37] introduce additional spin-dependent two-body terms and/or extra velocitydependent three-body contributions. The three-body force is replaced in other approaches by a more general two-body density-dependent term including usually some power of the density. An example for this type of extended interaction is the forces of Van Giai and Sagawa, SG1 and SG2 [17], where a fractional power of the density was introduced. In addition to the ground-state properties, these forces give also quite reasonable descriptions of the Gamow-Teller resonances and are free of spin instabilities. These latter forces are therefore of particular interest to study the spin $M 1$ excitations, and are considered here.

The two-body density-dependent Skyrme forces used here have the structure,

$$
\begin{aligned}
V\left(\mathbf{r}_{1}, \mathbf{r}_{2}\right)= & t_{0}\left(1+x_{0} P_{\sigma}\right) \delta\left(\mathbf{r}_{1}-\mathbf{r}_{2}\right)+\frac{1}{2} t_{1}\left(1+x_{1} P_{\sigma}\right)\left(k^{2}+k^{\prime 2}\right) \delta\left(\mathbf{r}_{1}-\mathbf{r}_{2}\right)+t_{2}\left(1+x_{2} P_{\sigma}\right) \mathbf{k}^{\prime} \cdot \delta\left(\mathbf{r}_{1}-\mathbf{r}_{2}\right) \mathbf{k} \\
& +\frac{1}{6} t_{3} \rho^{\alpha}\left(1+x_{3} P_{\sigma}\right) \delta\left(\mathbf{r}_{1}-\mathbf{r}_{2}\right)+i W \mathbf{k}^{\prime} \cdot \delta\left(\mathbf{r}_{1}-\mathbf{r}_{2}\right)(\boldsymbol{\sigma} \times \mathbf{k}),
\end{aligned}
$$

where $\mathbf{k}=(2 i)^{-1}\left(\mathbf{k}_{1}-\mathbf{k}_{2}\right)$ and $\mathbf{k}^{\prime}=-(2 i)^{-1}\left(\mathbf{k}_{1}^{\prime}-\mathbf{k}_{2}^{\prime}\right)$ operate to the right and left, respectively. $P_{\sigma}$ is the spin exchange operator and $t_{0}, t_{1}, t_{2}, t_{3}, x_{0}, x_{1}, x_{2}, x_{3}, W, \alpha$ are parameters fitted to nuclear ground-state properties. The values of these parameters for the various forces used in this work are listed in Table I. All of these forces except Sk3 [31] are general- ized Skyrme forces in the sense that they contain more involved density dependences.

\section{B. RPA and residual interactions}

The model Hamiltonian used here to solve the RPA equations for the $I^{\pi} K=1^{+} 1$ excitations has the form 


$$
H=H_{0}+H_{\mathrm{QQ}}+H_{\mathrm{SS}}+H_{\mathrm{RV}} .
$$

It contains a deformed mean field $H_{0}$ with BCS correlations and separable quadrupole-quadrupole $\left(H_{\mathrm{QQ}}\right)$, spin-spin $\left(H_{\mathrm{SS}}\right)$, and rotation-vibration $\left(H_{\mathrm{RV}}\right)$ residual interactions. The structure of $H$ is similar to that in our previous works $[13,14,20]$.

We sketch in this subsection the main ingredients of our RPA formalism based on the model Hamiltonian (2). We solve the RPA equations

$$
\begin{gathered}
{\left[H, \Gamma^{\dagger}(\nu m)\right]=W_{\nu} \Gamma^{\dagger}(\nu m),} \\
{\left[\Gamma(\nu m), \Gamma^{\dagger}\left(\nu^{\prime} m^{\prime}\right)\right]=\delta\left(\nu, \nu^{\prime}\right) \delta\left(m, m^{\prime}\right),}
\end{gathered}
$$

in the deformed HF basis. In these equations $W_{\nu}$ is the excitation energy of the corresponding RPA state, generated by the RPA phonon creation operator $\Gamma^{\dagger}(\nu m)$. The explicit expressions of the residual separable forces $\left(H_{\mathrm{QQ}}, H_{\mathrm{SS}}, H_{\mathrm{RV}}\right)$ used to describe $I^{\pi} K=1^{+} 1$ excitations can be found in our previous publications [see, for instance, Eqs. (2)-(4) in Ref. [14]].

The coupling constants in $H_{\mathrm{QQ}}$ and $H_{\mathrm{RV}}$ are selfconsistently determined from the condition of rotational invariance following a method described in detail in Ref. [18]. First, the coupling constants of $H_{\mathrm{QQ}}$ are determined in the quasiboson approximation by the condition $\left[H_{0}+H_{\mathrm{QQ}}, J(m)\right] \approx 0$, inspired by the work of Baznat and Pyatov [39]. Then, to ensure that $\left[H_{\mathrm{RPA}}, J(m)\right]=0$ is fulfilled exactly, we impose the constraint

$$
\left\langle\left[J(m), \Gamma^{\dagger}\left(\nu m^{\prime}\right)\right]\right\rangle=0 .
$$

The coupling constants of $H_{\mathrm{RV}}$ are determined microscopically by this constraint which ensures the orthogonality of the RPA wave functions to the spurious rotational state. Hence, the coupling constants of $H_{\mathrm{QQ}}$ and $H_{\mathrm{RV}}$ are derived from the deformed mean field and, therefore, they depend on the Skyrme force used to get the self-consistent mean field. However, the coupling constants of $H_{\mathrm{SS}}$ are not constrained by the above conditions.

In Refs. $[13,14,20]$ we studied the role of the different residual interactions and their influence on the different types of $K^{\pi}=1^{+}$excitations using a Woods-Saxon for the deformed field. We found the spin-spin interaction to be the most important one to describe spin excitations and spin $M 1$ strength distributions. In those works the spin-spin interaction was not connected to the mean field but its coupling strengths were taken from nuclear matter calculations. A review of possible choices for the coupling constants of the $H_{\text {SS }}$ interaction $\left(K_{S}\right.$ and $\left.q\right)$ can be found in [19]. In what follows we derive the coupling constants of the spin-spin residual interaction consistently from the same Skyrme force used to generate the mean field.

Following the standard procedure [16], we start from the Hartree-Fock ground state. The spin-dependent particle-hole residual interaction is defined in terms of second derivatives of the Hamiltonian density $H(\rho)$ with respect to the onebody densities $\rho_{s t}$,

$$
\begin{aligned}
V_{\mathrm{ph}}= & \delta\left(\mathbf{r}_{1}-\mathbf{r}_{2}\right) \frac{1}{16} \sum_{s t s^{\prime} t^{\prime}}\left[(-1)^{s-s^{\prime}} \boldsymbol{\sigma}_{1} \cdot \boldsymbol{\sigma}_{2}\right. \\
& \left.+(-1)^{s-s^{\prime}+t-t^{\prime}} \boldsymbol{\sigma}_{1} \cdot \boldsymbol{\sigma}_{2} \boldsymbol{\tau}_{1} \cdot \boldsymbol{\tau}_{2}\right] \frac{\delta^{2} H(\rho)}{\delta \rho_{s t} \delta \rho_{s^{\prime} t^{\prime}}}
\end{aligned}
$$

where $s$ and $t$ are the third components of the spin and isospin. This particle-hole interaction can be written in the language of the Landau-Migdal theory of Fermi systems [40], and a relationship can be established [17,30,32,34] between the Landau and Skyrme parameters in nuclear matter. For completeness we write here the expressions for the Landau parameters $G_{0}$ and $G_{0}^{\prime}$ corresponding to the two-body density-dependent Skyrme forces in Eq. (1):

$$
\begin{aligned}
G_{0}= & -N_{0}\left\{\frac{1}{4} t_{0}\left(1-2 x_{0}\right)+\frac{1}{24} t_{3} \rho^{\alpha}\left(1-2 x_{3}\right)\right. \\
& \left.+\frac{1}{8} k_{F}^{2}\left[t_{1}\left(1-2 x_{1}\right)-t_{2}\left(1+2 x_{2}\right)\right]\right\}, \\
G_{0}^{\prime}= & -N_{0}\left[\frac{1}{4} t_{0}+\frac{1}{24} t_{3} \rho^{\alpha}+\frac{1}{8} k_{F}^{2}\left(t_{1}-t_{2}\right)\right] .
\end{aligned}
$$

We recall here that $G_{0}$ and $G_{0}^{\prime}$ are the Landau parameters for the isoscalar and isovector spin-spin interactions, respectively. We also note that the $t_{3}$ term in Sk3 can be written as a contact three-body force that leads to a different dependence of the Landau parameters on the parameter $t_{3}$.

Finally, in order to find the coupling constants $K_{S}$ and $q$ of $H_{\mathrm{SS}}$, we have to relate the contact Landau-Migdal force with the separable spin-spin interaction [Eq. (3) in Ref. [14]]. Assuming a constant density distribution inside a sphere with radius $R=r_{0} A^{1 / 3}\left(r_{0}=1.3 \mathrm{fm}\right)$, we integrate the LandauMigdal interaction over this volume [19]. This procedure provides a connection between the parameters of our separable spin-spin force $K_{S}$ and $q$ and the Landau parameters, and therefore connects $K_{S}$ and $q$ with the Skyrme parameters. In this way we obtain, for the overall strength $K_{S}$ of $H_{\text {SS }}$, the relation

$$
K_{S}=8 \frac{3}{4 \pi r_{0}^{3}} N_{0}^{-1}\left(G_{0}+G_{0}^{\prime}\right)
$$

and, for the ratio $q$ of the $n-p$ to the $n-n$ (or $p-p$ ) strengths in $H_{\mathrm{SS}}$, the relation

$$
q=\left(G_{0}-G_{0}^{\prime}\right) /\left(G_{0}+G_{0}^{\prime}\right) .
$$

Clearly, $q=+1(-1)$ corresponds to a purely isoscalar (isovector) $H_{\mathrm{SS}}$ interaction.

We list in Table II the values of the saturation density $\rho$, Fermi momentum $k_{F}$, effective mass $m^{*}$, and the normalization coefficient $N_{0}^{-1}$ for various Skyrme forces used in this work, as well as the corresponding values for the Landau parameters $G_{0}$ and $G_{0}^{\prime}$, Eq. (7), and the spin-spin parameters of the separable interaction $K_{S}$, Eq. (8), and $q$, Eq. (9). In the case of Sk3 we also give within parentheses the values obtained when the three-body version of the force is used.

One may question how reliable the separable force in comparison with the Landau-Migdal interaction for studying spin $M 1$ excitations is. This point has been considered in Ref. [41], where a comparison has been made for ${ }^{154} \mathrm{Sm}$ between the spin $M 1$ strength distributions obtained from a 
TABLE II. Saturation density $\rho$, Fermi momentum $k_{F}$, effective mass $m^{*}$, normalization coefficient $N_{0}^{-1}$, Landau parameters $G_{0}$ and $G_{0}^{\prime}$, Eq. (7), and the coupling strengths $K_{S}$, Eq. (8), and $q$, Eq. (9), of the separable spin-spin residual interaction derived from various Skyrme interactions through the Landau-Migdal force. Values within parentheses correspond to the three-body version of Sk3.

\begin{tabular}{lcccccccc}
\hline \hline & $\begin{array}{c}\rho \\
{\left[\mathrm{fm}^{-3}\right]}\end{array}$ & $\begin{array}{c}k_{F} \\
{\left[\mathrm{fm}^{-1}\right]}\end{array}$ & $m^{*} / m$ & $\begin{array}{c}N_{0}^{-1} \\
{\left[\mathrm{MeV} \mathrm{fm}^{3}\right]}\end{array}$ & $G_{0}$ & $G_{0}^{\prime}$ & $\begin{array}{c}K_{S} \\
{[\mathrm{MeV}]}\end{array}$ & $q$ \\
\hline SG2 [17] & 0.158 & 1.328 & 0.786 & 196.0 & 0.013 & 0.505 & 88 & -0.95 \\
SG1 [17] & 0.154 & 1.317 & 0.608 & 255.5 & 0.072 & 0.502 & 128 & -0.75 \\
Sk3 [31] & 0.145 & 1.291 & 0.763 & 207.8 & 0.052 & 0.460 & 92 & -0.80 \\
& & & & & $(-1.576)$ & $(-0.354)$ & $(-349)$ & $(0.60)$ \\
G $_{\sigma}[36]$ & 0.158 & 1.326 & 0.784 & 196.8 & -0.099 & 0.463 & 62 & -1.54 \\
Ska [33] & 0.155 & 1.320 & 0.608 & 254.7 & -0.015 & 0.324 & 68 & -1.10 \\
Skm* [35] & 0.160 & 1.334 & 0.788 & 194.5 & -0.297 & 0.315 & 3 & -34.0 \\
\hline \hline
\end{tabular}

contact interaction and a corresponding separable spin-spin force. The very similar results obtained have led to the conclusion [41] that, although less realistic, the separable interaction contains the essential features of the zero-range force. An important advantage of using separable interactions is that one can avoid the drastic basis cutoff necessary for heavy deformed nuclei when using a Landau-Migdal force. Our basis includes all the configurations with $K^{\pi}=1^{+}$and two-quasiparticle energies up to $30 \mathrm{MeV}$, i.e., about 1500 irreducible two-quasiparticle configurations in each rareearth nucleus.

\section{MEAN-FIELD PROPERTIES WITH VARIOUS SKYRME FORCES}

As already mentioned, except for the three-body version of SK3, all the Skyrme forces studied in this work are twobody density-dependent interactions of the type (1). Their parameters are listed in Table I. The force Sk3 [31], representative of the first generation Sk1-Sk6, is a simple one and usually regarded as a standard reference. The remaining interactions in Table I are generalizations including a fractional power of the density, $\alpha<1$ in Eq. (1). They are designed to fit more extensive sets of data. All the Skyrme forces in Table I lead to similar ground-state properties (binding energies, rms radii, etc.) In particular, the SG forces [17] are of special interest for our present study since they provide a reasonable description of Gamow-Teller strength distributions. The intrinsic quadrupole moments and rms radii of the nuclei considered here obtained from SG2 agree also very well with the corresponding experimental data from Refs. $[42,43]$ as one can see from Table III.

All the results presented in this paper have been obtained using effective spin gyromagnetic factors $g_{s}^{\text {eff }}=0.7 g_{s}^{\text {free }}$. By doing this we account in an effective way for the quenching of the $M 1$ strength caused by additional degrees of freedom not considered here.

The unperturbed $\left(H_{\mathrm{SS}}=0\right)$ spin $M 1$ strength distributions obtained with the various Skyrme interactions from Table I are plotted in Fig. 1 in the example of ${ }^{154} \mathrm{Sm}$. In the absence of a spin-spin residual interaction, the spin $M 1$ strength distribution originates almost exclusively from the mean field. The spin $M 1$ distribution is rather insensitive to the other residual interactions $H_{\mathrm{QQ}}$ and $H_{\mathrm{RV}}$ and it is close to the particle-hole strength distribution. Also, for comparison, we include in Fig. 1 the results obtained from a deformed Woods-Saxon potential with the parametrization of Tanaka et al. [44] used in Refs. [13,14,20]. In this figure, as well as in the remaining figures to be discussed later, the strength $B_{\sigma}(M 1)$ is summed over energy bins of $80 \mathrm{keV}$ to facilitate the comparison to experiment. We have folded the theoretical strength distributions with Gaussians whose widths are energy dependent. This has been done to simulate the further fragmentation of the strength expected from the coupling to four-quasiparticle states not taken into account in the RPA. Since the density of these states increases with energy [45], the widths of our Gaussians increase linearly from $\Gamma=0.5$ $\mathrm{MeV}$ at $E=4 \mathrm{MeV}$ to $\Gamma=1.5 \mathrm{MeV}$ at $E=12 \mathrm{MeV}$ in a way similar to other works [21-23]. The microscopic origin of these widths has been studied in Ref. [46].

The purpose of Fig. 1 is to compare the effect of the different mean fields on the spin $M 1$ strength distributions before including the spin-spin residual interaction.

There is a remarkable difference between the results obtained with self-consistent and Woods-Saxon mean fields. The spin $M 1$ strength is distributed in the former case over an extended energy range, while in the latter case the strength is concentrated in a narrower range. One can see that the profile of the spin $M 1$ strength distribution is rather similar for all the Skyrme interactions considered. The main difference is that the strength is displaced in energy for the different Skyrme interactions. This is due to the spin-orbit force. The position of the peaks is strongly correlated with the value of the spin-orbit parameter $W(1)$, shown in Table I where the Skyrme forces are listed in ascending order of their $W$ values. The larger is $W$, the more shifted to higher energy the $M 1$ strength is. That the spin-orbit force plays an important role in the spin $M 1$ strength distribution was also noticed in Refs. $[14,20]$ in the context of the Woods-Saxon potential. The strongest peak located around $6 \mathrm{MeV}$ contains much more strength than experimentally observed (see Fig. 2). These qualitative features are common for all the nuclei considered in this paper. They also hold for other Skyrme interactions of the type (1) not shown here.

The detailed structure of the unperturbed spin excitations obtained with SG2 is shown in Fig. 2 as an example. The same folded distribution as in Fig. 1 is displayed in the top plot together with the experimental data [7]. One can see in the middle plot of Fig. 2 the individual spin $M 1$ excitations, 
TABLE III. Intrinsic quadrupole moments $Q_{0}$ and root-mean-square charge radii calculated from the HF mean field with the force SG2 including BCS with fixed pairing gaps $\left(\Delta_{p}=\Delta_{n}=1 \mathrm{MeV}\right.$ for the rare earths and $\Delta_{p}=\Delta_{n}=0.7 \mathrm{MeV}$ for the actinides). They are compared with the experimental values from Refs. [42] and [43], respectively. The theoretical RPA spin $M 1$ strengths summed up to $12 \mathrm{MeV}$ and the corresponding energy-weighted sums are listed in the two last columns.

\begin{tabular}{|c|c|c|c|c|c|c|}
\hline Nucleus & $\begin{array}{c}Q_{0}^{\text {th }} \\
{\left[\begin{array}{ll}e & \mathrm{~b}\end{array}\right]}\end{array}$ & $\begin{array}{l}Q_{0}^{\operatorname{expt}} \\
{\left[\begin{array}{ll}e & \mathrm{~b}\end{array}\right]}\end{array}$ & $\begin{array}{c}\left\langle r^{2}\right\rangle_{\text {th }}^{1 / 2} \\
{[\mathrm{fm}]}\end{array}$ & $\begin{array}{c}\left\langle r^{2}\right\rangle_{\text {expt }}^{1 / 2} \\
{[\mathrm{fm}]}\end{array}$ & $\begin{array}{c}\sum_{\nu} B_{\sigma}(M 1, \nu) \\
{\left[\mu_{N}^{2}\right]}\end{array}$ & $\begin{array}{c}\Sigma_{\nu} E_{\nu} B_{\sigma}(M 1, \nu) \\
{\left[\mathrm{MeV} \mu_{N}^{2}\right]}\end{array}$ \\
\hline${ }^{146} \mathrm{Nd}$ & 2.772 & $2.760(50)$ & 4.966 & 4.970 & 8.2 & 58.8 \\
\hline${ }^{148} \mathrm{Nd}$ & 3.720 & $3.725(40)$ & 5.006 & 5.002 & 8.9 & 64.4 \\
\hline${ }^{150} \mathrm{Nd}$ & 4.953 & $5.258(38)$ & 5.053 & 5.048 & 10.2 & 77.3 \\
\hline${ }^{150} \mathrm{Sm}$ & 4.013 & $3.684(41)$ & 5.028 & $5.045(6)$ & 9.0 & 64.1 \\
\hline${ }^{152} \mathrm{Sm}$ & 5.577 & $5.881(34)$ & 5.080 & $5.093(6)$ & 10.5 & 80.3 \\
\hline${ }^{154} \mathrm{Sm}$ & 6.624 & $6.620(38)$ & 5.133 & $5.126(8)$ & 11.4 & 92.8 \\
\hline${ }^{156} \mathrm{Gd}$ & 6.773 & $6.830(37)$ & 5.145 & 5.068 & 11.5 & 90.7 \\
\hline${ }^{158} \mathrm{Gd}$ & 7.068 & $7.104(35)$ & 5.174 & $5.172(6)$ & 11.8 & 94.3 \\
\hline${ }^{160} \mathrm{Gd}$ & 7.274 & $7.265(42)$ & 5.200 & - & 11.9 & 95.6 \\
\hline${ }^{160} \mathrm{Dy}$ & 7.234 & $7.13(10)$ & 5.190 & - & 11.4 & 89.1 \\
\hline${ }^{162} \mathrm{Dy}$ & 7.460 & $7.28(10)$ & 5.216 & - & 12.2 & 93.9 \\
\hline${ }^{164}$ Dy & 7.635 & $7.503(34)$ & 5.242 & - & 12.2 & 95.1 \\
\hline${ }^{166} \mathrm{Er}$ & 7.804 & $7.656(33)$ & 5.258 & $5.237(16)$ & 12.2 & 94.6 \\
\hline${ }^{168} \mathrm{Er}$ & 7.929 & $7.630(60)$ & 5.282 & - & 12.4 & 98.0 \\
\hline${ }^{170} \mathrm{Er}$ & 7.931 & $7.65(6)$ & 5.302 & - & 12.7 & 101.8 \\
\hline${ }^{172} \mathrm{Yb}$ & 8.116 & $7.792(45)$ & 5.319 & - & 12.8 & 102.8 \\
\hline${ }^{174} \mathrm{Yb}$ & 7.937 & $7.728(39)$ & 5.336 & $5.41(3)$ & 13.0 & 103.5 \\
\hline${ }^{176} \mathrm{Yb}$ & 7.714 & 7.37(7) & 5.353 & 5.443 & 13.0 & 102.6 \\
\hline${ }^{182} \mathrm{~W}$ & 6.909 & $6.46(8)$ & 5.393 & - & 12.5 & 92.9 \\
\hline${ }^{184} \mathrm{~W}$ & 6.657 & $6.12(6)$ & 5.409 & $5.42(7)$ & 12.7 & 96.4 \\
\hline${ }^{186} \mathrm{~W}$ & 6.347 & $5.88(5)$ & 5.423 & $5.40(4)$ & 12.9 & 97.3 \\
\hline${ }^{232} \mathrm{Th}$ & 9.201 & $9.659(47)$ & 5.826 & 5.7723 & 14.9 & 106.9 \\
\hline${ }^{236} \mathrm{U}$ & 10.586 & $10.80(7)$ & 5.870 & - & 15.2 & 112.3 \\
\hline${ }^{238} \mathrm{U}$ & 10.635 & 11.02(9) & 5.885 & 5.854 & 15.1 & 110.8 \\
\hline
\end{tabular}

revealing better the structure hidden behind the folding procedure.

The ratio $R_{i v}$,

$$
R_{i v}=\frac{S_{i v}^{2}}{S_{i s}^{2}+S_{i v}^{2}}, \quad S_{i v}^{i s}=\left\langle 1_{\nu}^{+}\left|S_{n}\right|\right\rangle \pm\left\langle 1_{\nu}^{+}\left|S_{p}\right|\right\rangle,
$$

defined as in Ref. [14], is shown for each spin excitation with $B(M 1)>0.2 \mu_{N}^{2}$ in the bottom plot of the figure. This ratio is 1 (0) for purely isovector (isoscalar) excitations. A value $R_{i v}=0.5$ indicates a pure proton or neutron excitation, i.e., a mixed isoscalar and isovector character. The structure of the excitations, revealed by analyzing the $R_{i v}$ values, has a mixed character with values scattered around the pure proton or neutron excitation $\left(R_{i v}=0.5\right)$.

\section{ROLE OF THE RESIDUAL SPIN-SPIN INTERACTION}

The spin-spin residual interaction can produce substantial redistribution of the spin $M 1$ strength, while the remaining residual interactions $H_{\mathrm{QQ}}$ and $H_{\mathrm{RV}}$ have a negligible influence on it. In this section we study the effect of the spin-spin residual interaction $H_{\text {SS }}$ derived from the various Skyrme forces. We focus this study on the two-body densitydependent Skyrme forces. The effect of $H_{\mathrm{SS}}$ can be best seen by comparing Figs. 2 and 3. The theoretical results in the presence of the spin-spin residual interaction are shown in Fig. 3 for the same nucleus ${ }^{154} \mathrm{Sm}$ and the same Skyrme force SG2, as in Fig. 2. The coupling constants of $H_{\mathrm{SS}}$ are in this case $K_{S}=88 \mathrm{MeV}$ and $q=-0.95$ (see Table II).

Comparison between the upper two plots of Figs. 2 and 3 demonstrates that the excessive unperturbed strength below 7 $\mathrm{MeV}$ in Fig. 2 is moved to higher energies when $H_{\mathrm{Ss}}$ is switched on in Fig. 3. The agreement with experiment is improved substantially by inclusion of the residual interaction $H_{\mathrm{SS}}$. This is due to the predominantly isovector character $(q \approx-1)$ of the $H_{\mathrm{SS}}$ interaction, derived from the SG2 force. This repulsive interaction moves the isovector strength to higher energy, as revealed by the comparison of the bot- 


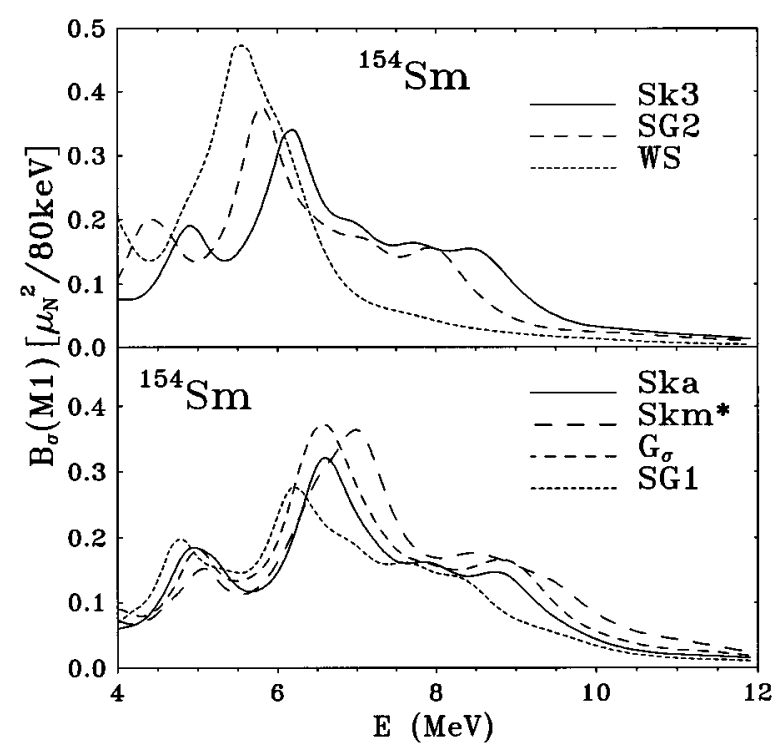

FIG. 1. Unperturbed $\left(H_{\mathrm{SS}}=0\right)$ energy distribution of the spin $M 1$ strength with $K^{\pi}=1^{+}$in ${ }^{154} \mathrm{Sm}$ for various Skyrme interactions from Table I. Also shown for comparison is the result obtained with the Woods-Saxon (WS) potential used in Ref. [14]. The units are $\mu_{N}^{2} / 80 \mathrm{keV}$ and the curves have been folded with Gaussians, whose widths increase linearly from $\Gamma=0.5 \mathrm{MeV}$ at $E=4 \mathrm{MeV}$ to $\Gamma=1.5 \mathrm{MeV}$ at $E=12 \mathrm{MeV}$.

tom plots in Figs. 2 and 3, where the ratio $R_{i v}$, Eq. (10), is shown only for excitations with $B_{\sigma}(M 1)>0.2 \mu_{N}^{2}$ One can see in Table II that all the Skyrme forces considered, except the three-body version of Sk3, share this common feature of a repulsive character for the isovector spin-spin interaction.

One can learn from the ratio $R_{i v}$ that the peak at $6 \mathrm{MeV}$ in Fig. 3 has an isoscalar character $\left(R_{i v}<0.2\right)$. The broader peak at higher energies in Fig. 3 is built up from excitations with a clearly marked isovector nature $\left(R_{i v}>0.7\right)$. In summary, the spin-spin residual interaction shifts the strength to higher energies and reinforces the isoscalar and isovector character of the lower- and higher-lying peaks, respectively. The position and strength of the two peaks agree well with experiment.

Spin $M 1$ strength distributions in rare-earth and actinide nuclei were studied in Refs. $[13,14,20]$ within a QRPA approach using a deformed Woods-Saxon potential and separable residual interactions. In particular, the two peaks were obtained for ${ }^{154} \mathrm{Sm}$ at the right position, the lower one containing less strength than experiment and the higher one with the correct strength. The two peaks were interpreted as isoscalar and isovector. Spin $M 1$ strength distributions in rareearth nuclei have also been studied in other microscopic calculations. Results within the Tamm-Dancoff approximation using a deformed Nilsson potential, quadrupole-quadrupole, spin-spin, and Coriolis residual interactions were presented in Ref. [23]. The peaks of the spin $M 1$ strength distribution were interpreted there as corresponding to proton or neutron spin-flip configurations. A similar interpretation was proposed in Ref. [24] within the QRPA using a deformed Woods-Saxon potential with separable residual interactions. The absence of substantial neutron-proton mixing is due to the small $q$ value, common to these works. This means an almost vanishing neutron-proton spin-spin residual interac-

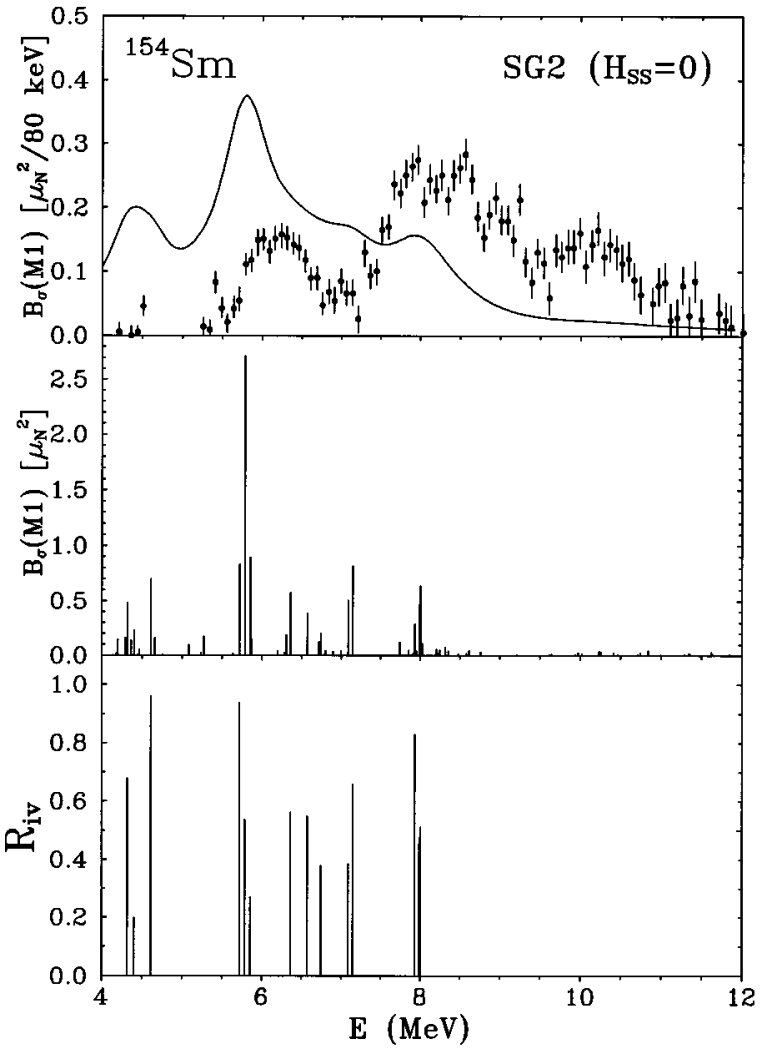

FIG. 2. Top plot: unperturbed $\left(H_{\mathrm{SS}}=0\right)$ energy distribution of the spin $M 1$ strength with $K^{\pi}=1^{+}$obtained with the force SG2 in ${ }^{154} \mathrm{Sm}$. Units and Gaussian widths of the theoretical curve (solid line) are as in Fig. 1. Experimental data (dots with error bars) are from Ref. [7]. Middle plot: spin $M 1$ spectrum of individual excitations before the folding procedure. Bottom plot: Spin isovector ratio (10) for the excitations with $B_{\sigma}(M 1)>0.2 \mu_{N}^{2}$.

tion. QRPA results for $M 1$ spin strength distributions have also been reported in Ref. [21,22]. They were obtained with a deformed Woods-Saxon potential and a zero-range Landau-Migdal residual interaction. The first peak of ${ }^{154} \mathrm{Sm}$ at around $6 \mathrm{MeV}$ was overestimated in [22-24] by a factor of about 2 and the higher-energy peak at around $8 \mathrm{MeV}$ was also overestimated in Ref. [22].

From Figs. 2 and 3 and the analysis made in terms of the ratios $R_{i v}$, one can see that in the unperturbed case the interpretation of the peaks is compatible with that of Ref. [23]; i.e., the peaks are built by predominantly proton or neutron spin $M 1$ excitations. Nevertheless, in the full calculation when the self-consistent residual spin-spin interaction is active, we recover our former interpretation $[13,14,20]$ for the predominantly isoscalar or isovector nature of the two peaks.

Theoretical QRPA results obtained with the various twobody Skyrme forces from Table I are displayed in Fig. 4 and compared to experimental data for the same nucleus ${ }^{154} \mathrm{Sm}$. Results obtained from a Woods-Saxon potential and a phenomenological spin-spin interaction with $K_{S}=200 \mathrm{MeV}$ and $q=-0.5[14]$ are also plotted for comparison (short-dashed curve in the upper plot). Looking at Fig. 4 one can see that the structure of the strength distribution is qualitatively similar for all the Skyrme forces considered. This resembles the situation for the unperturbed strength in Fig. 1. In spite of the fact that the Skyrme forces studied lead to different coupling 


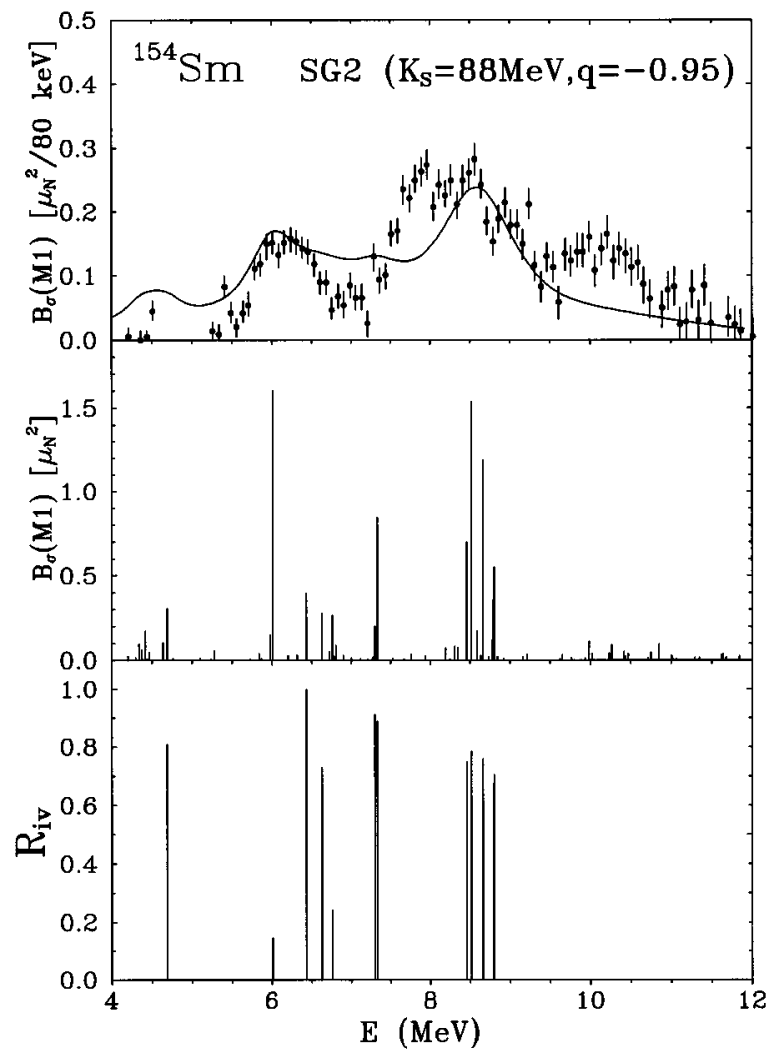

FIG. 3. Same as in Fig. 2 but with the self-consistent residual spin-spin interaction $H_{\mathrm{SS}}$ included. Its coupling constants are listed in the first row of Table II.

constants $K_{S}$ and $q$, the values of the isovector coupling constants $G_{0}^{\prime}$ are close to each other (see Table II). The isoscalar coupling constant $G_{0}$ is about one order of magnitude smaller than $G_{0}^{\prime}$, except for $\mathrm{Skm}^{*}$. The value of $G_{0}$ affects the isoscalar strength distribution whose contribution to $B_{\sigma}(M 1)$ is negligible because of the very small value of the

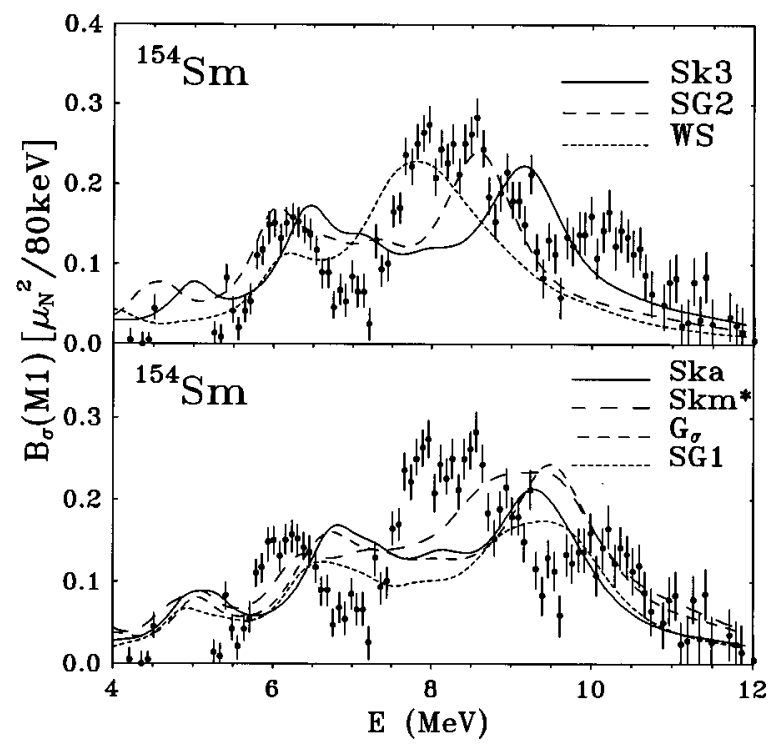

FIG. 4. Same as in Fig. 1 but with the self-consistent residual spin-spin interactions $H_{\mathrm{SS}}$ included. In the case of the Sk3 force, $H_{\mathrm{SS}}$ is obtained from the two-body version. isoscalar spin gyromagnetic factor. We recall here that the suppression of the isoscalar spin strength with respect to the isovector one is dictated by the Morpurgo factor [47]

$$
\frac{\left(g_{s}^{n}+g_{s}^{p}\right)^{2}}{\left(g_{s}^{n}-g_{s}^{p}\right)^{2}} \sim \frac{1}{30}
$$

Thus, the effect of $H_{\mathrm{SS}}$ on the spin $M 1$ strength distribution is determined mainly from its isovector part. Hence, the shift of isovector strength to higher energies, discussed above for SG2, takes place in the same way for the other Skyrme twobody forces considered, because the values of $G_{0}^{\prime}$ are similar.

In addition, comparison of the values of $W$ in Table I and $G_{0}^{\prime}$ in Table II demonstrates that a stronger shift to higher energy produced by a stronger spin-orbit interaction (larger $W$ ) is compensated to some extent by the smaller shift produced by a smaller value of $G_{0}^{\prime}$. This explains why the results with different Skyrme forces in Fig. 4 are so similar. Nevertheless, the SG2 force seems to provide a better overall description of experimental data when the above comparison of various Skyrme two-body forces is extended to the whole set of nuclei considered here.

It is interesting to remark that the strength of the spin-spin residual interaction obtained from the two-body Skyrme forces is significantly smaller than that of the phenomenological $H_{\text {SS }}$ interaction needed when the Woods-Saxon potential is used. This reflects the fact that the self-consistent HF mean field, derived from the two-body Skyrme forces, contains already an effect from the Skyrme spin-spin interaction and $H_{\text {SS }}$ behaves truly as a residual (weak) interaction, while this effect is not contained in the Woods-Saxon potential.

\section{COMPARISON WITH EXPERIMENT}

In this section we compare to experiment the theoretical results obtained with the SG2 force. This comparison can be seen in Figs. 5-8 covering all the nuclei for which experimental data are available. The unperturbed $\left(H_{\mathrm{SS}}=0\right)$ spin $M 1$ strength distribution is displayed in each plot by a shortdashed curve, while that obtained with the consistent spinspin residual interaction from Table II $\left(K_{S}=88 \mathrm{MeV}\right.$, $q=-0.95)$ is shown with a solid line. As is well known, the energy splitting of the HF states around the Fermi level is too large. This has an effect on the spin $M 1$ distributions that can be roughly compensated by reducing the $K_{S}$ value. To illustrate this effect, we show in Figs. 5-8 by long-dashed lines the results corresponding to a purely isovector spin-spin residual interaction $(q=-1)$ whose strength $K_{S}=50 \mathrm{MeV}$ has been slightly decreased with respect to the original selfconsistent value $K_{S}=88 \mathrm{MeV}$. The main effect from this reduction is a slight shift of the second bump to lower energy, which improves in some nuclei the agreement with experiment.

The results for the rare earths are shown in Fig. 5 for ${ }^{150} \mathrm{Nd}$, ${ }^{154} \mathrm{Sm}$, and in Fig. 6 for ${ }^{156,158} \mathrm{Gd}$ and ${ }^{168} \mathrm{Er}$. The results for the actinides ${ }^{232} \mathrm{Th}$ and ${ }^{238} \mathrm{U}$ are displayed in Fig. 7. Finally, we show results for ${ }^{56} \mathrm{Fe}$ in Fig. 8. The experimental data exhibit in most nuclei a double-humped structure that is well described theoretically. The data for ${ }^{150} \mathrm{Nd}$ and ${ }^{154} \mathrm{Sm}$ in Fig. 7 exhibit a third bump beyond $10 \mathrm{MeV}$. It has 


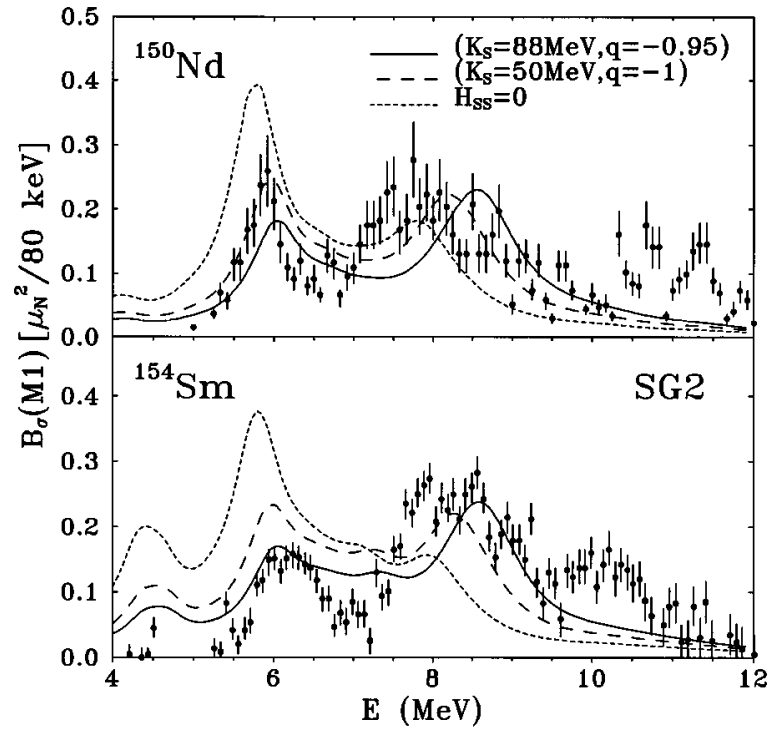

FIG. 5. Energy distribution of the spin $M 1$ strength with $K^{\pi}=1^{+}$obtained with the force SG2 in ${ }^{150} \mathrm{Nd}$ and ${ }^{154} \mathrm{Sm}$. Units and Gaussian widths are as in Fig. 1. Results obtained with the self-consistent spin-spin residual interaction (solid lines, $K_{S}=88$ $\mathrm{MeV}, q=-0.95$ from Table II), with the values $K_{S}=50 \mathrm{MeV}$, $q=-1$ (dashed lines), and with $H_{\mathrm{SS}}=0$ (short-dashed lines). Experimental data (dots with error bars) are from Refs. [7,12].

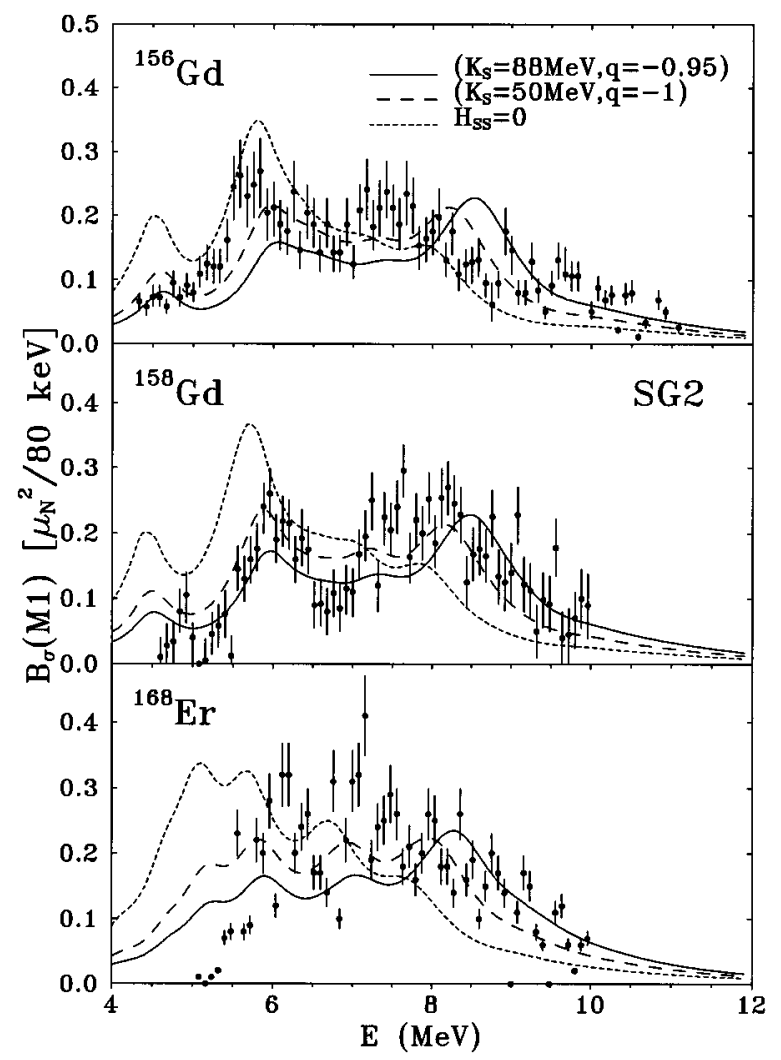

FIG. 6. Same as in Fig. 5 but for ${ }^{156,158} \mathrm{Gd}$ and ${ }^{168}$ Er. Experimental data (dots with error bars) are from Ref. $[8,12]$.

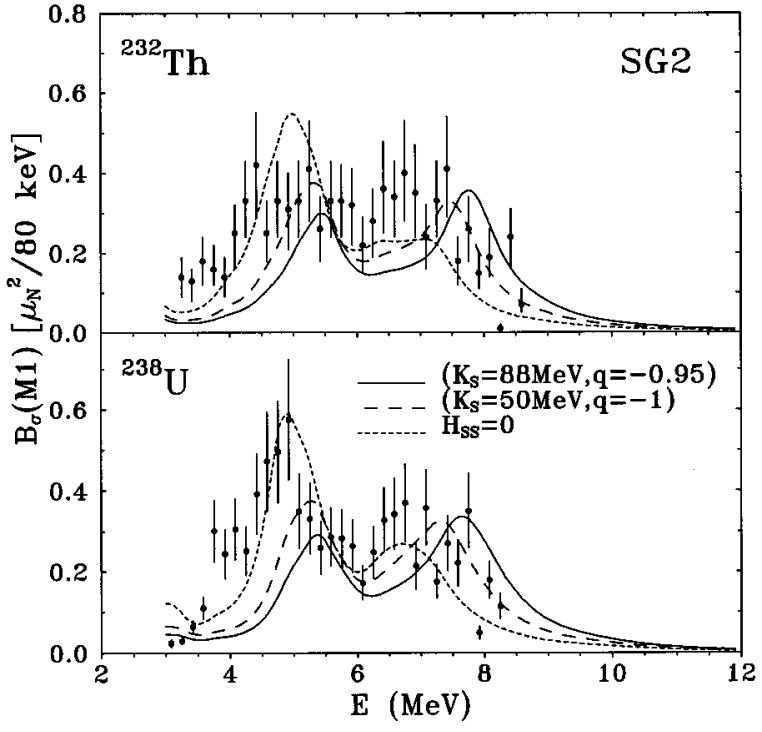

FIG. 7. Same as in Fig. 5 but for ${ }^{232} \mathrm{Th}$ and ${ }^{238} \mathrm{U}$. Experimental data (dots with error bars) are from Ref. [12].

been seen in recent analyses of $\left(p, p^{\prime}\right)$ experiments which are still preliminary. A survey of Figs. 5-8 indicates that only the small experimental strength beyond $10 \mathrm{MeV}$ in ${ }^{150} \mathrm{Nd}$ and ${ }^{154} \mathrm{Sm}$ is missing in our theoretical description. A possible explanation for its origin could be that it arises from $\Delta K=0$ excitations not considered in this work. It was found in [41] that this strength is located beyond $8 \mathrm{MeV}$ and it is considerably smaller than the strength with $|\Delta K=1|$.

It is remarkable that the mean-field results contain already in most cases the basic features of the strength distributions, although the concentration of the strength below typically 7 $\mathrm{MeV}$ is larger than experimentally observed. In this respect, the spin-spin residual interaction acts in the right-direction moving part of this strength to higher energies. We note that the agreement with experiment after inclusion of the spinspin residual interaction is particularly improved in the case of ${ }^{154} \mathrm{Sm}$. This may be significant because the data for this nucleus, being measured with polarized protons, are more reliable.

We should also mention that the spin $M 1$ strengths summed up to $12 \mathrm{MeV}$ agree with the experimental values $[7,8,12]$. This is discussed in more detail in the next sections, where we also study energy-weighted sums and their characteristic features.

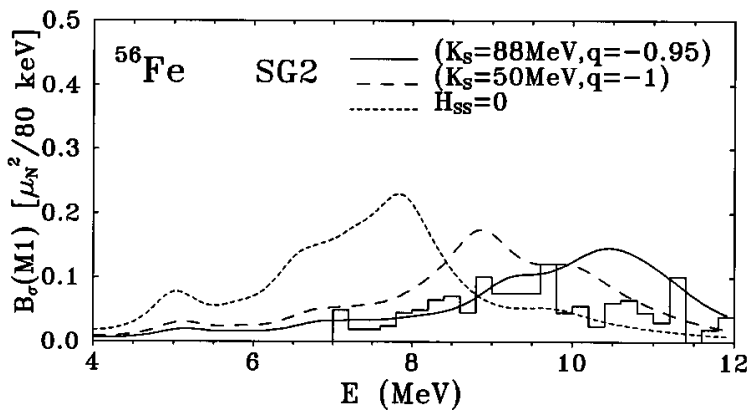

FIG. 8. Same as in Fig. 5 but for ${ }^{56} \mathrm{Fe}$. Experimental data (histograms) are from Ref. [7]. 


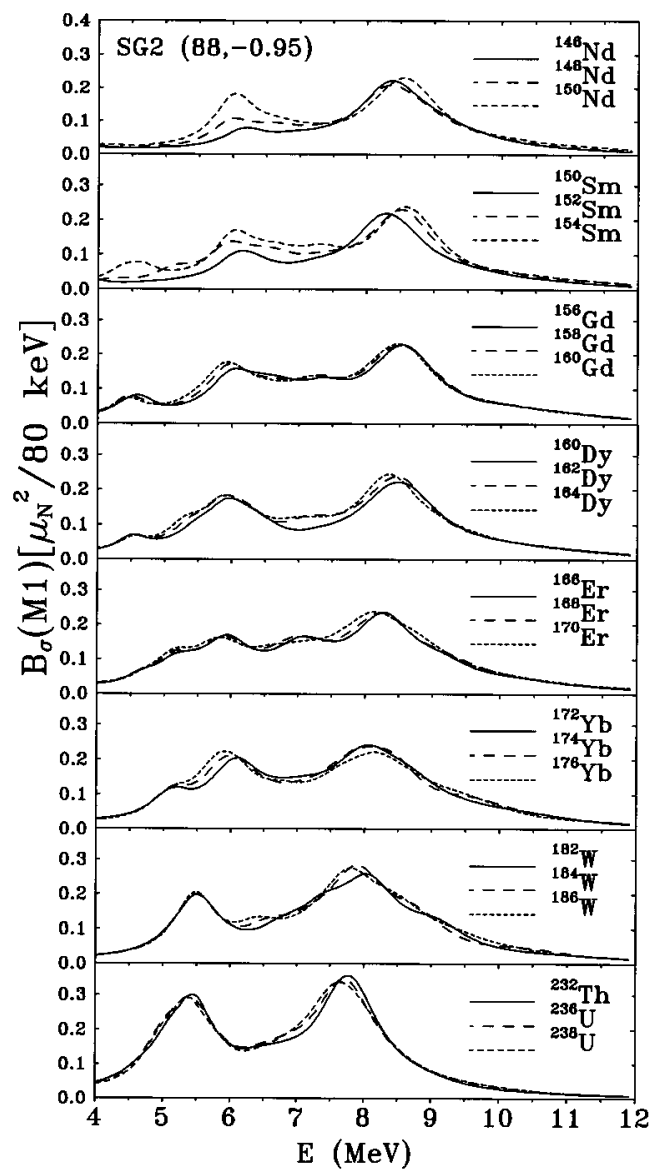

FIG. 9. Energy distributions of the spin $M 1$ strength with $K^{\pi}=1^{+}$obtained with the force SG2 for various rare-earth and actinide isotopes. Results obtained with the self-consistent spin-spin residual interaction $\left(K_{S}=88 \mathrm{MeV}, q=-0.95\right.$ from Table II). Units and Gaussian widths are as in Fig. 1.

\section{SYSTEMATICS OF THE SPIN M1 STRENGTH DISTRIBUTION}

The results shown in the previous sections give confidence in the predictive power of the method with the SG2 force when applying it to other, experimentally yet unexplored, nuclei. Results from systematic calculations in seven isotope chains from the rare-earth region and one chain of actinides are displayed in Fig. 9 and Table III.

In Table III we show the ground-state properties (quadrupole moments $Q_{0}$ and rms radii) of these nuclei together with their experimental values from Refs. [42,43]. Also shown in the two last columns are calculated summed spin $M 1$ strengths that will be discussed in the next section. As can be seen from Table III, the quadrupole moments and rms radii are well reproduced by the HF calculations with the SG2 force. The agreement is remarkable since no parameters have been used to fit these quantities in the present self-consistent approach, contrary to what was done in the past $[13,14]$ with Woods-Saxon potentials, where the deformation parameters of the potentials were chosen to reproduce the experimental quadrupole moments. It is well known that the calculations with Skyrme forces describe successfully [28] the groundstate properties of both spherical and deformed nuclei. Their ability to describe excited states and in particular unnaturalparity states is more questionable. Nevertheless, as already shown in the previous sections, the SG2 force describes successfully spin excitations.

In Fig. 9 we show the spin $M 1$ strength distributions obtained from our calculation. There is a common feature to all the nuclei from Fig. 9: the existence of two bumps, particularly pronounced in the actinides and in some of the rare earths. In the rare-earth region, the low-energy peak is located between 5 and $7 \mathrm{MeV}$ and the high-energy peak appears between 7 and $9 \mathrm{MeV}$. The two peaks are slightly shifted to lower energies in the actinide nuclei.

Richter made the observation [7] that the center of gravity of the excitation energy of the two experimentally observed peaks obeys a simple $A^{-1 / 3}$ law, characteristic for spin-flip excitations between shells of spin-orbit partners. Studying the dependence of the location of the peaks with the mass number in Fig. 9, we find that the excitation energy corresponding to the centroids of the two peaks is roughly proportional to $A^{-1 / 3}$, in agreement with experiment [7]. This approximate $A^{-1 / 3}$ law is also valid if one includes the nucleus ${ }^{56} \mathrm{Fe}$, which is far beyond the rare-earth and actinide regions.

In most cases there is a striking similarity of the spin $M 1$ strength profiles shown within a given isotope chain. This is true for the Gd, Dy, Er, Yb, W, and U isotopes. In these cases the intrinsic quadrupole moments of the isotopes considered within each chain are very close (see Table III). On the contrary, for the $\mathrm{Nd}$ and $\mathrm{Sm}$ isotope chains, the spin $M 1$ profiles shown vary substantially from one isotope to the other within each chain, and so do the quadrupole moments. This suggests that there is a correlation between the spin $M 1$ distributions of the $K^{\pi}=1^{+}$excitations and the quadrupole deformation. This point deserves further attention and will be discussed in the next section.

The energy-weighted and non-energy-weighted summed spin $M 1$ strengths are given in the two last columns of Table III. All the $K^{\pi}=1^{+}$excitations up to $12 \mathrm{MeV}$ have been included in the sums. The non-energy-weighted sums in Table III are in agreement with the available experimental information: $11 \pm 2 \mu_{N}^{2}[7,8]$ and $\sim 18 \mu_{N}^{2}$ [12] for the summed spin strength in rare-earths and actinides, respectively. A certain correlation of both energy-weighted and non-energy-weighted sums with the quadrupole moments can be observed in this table. It will be discussed in the next section.

\section{DEFORMATION DEPENDENCE}

The dependence of the spin $M 1$ strength distribution on the deformation had not been studied in detail until now. The topic was considered first by Zamick et al. [48] within a schematic model. It was concluded that the spin $M 1$ strength decreases with increasing deformation and vanishes in the large deformation limit for nuclei with only two neutrons and/or two protons outside closed shells. It should be stressed that this conclusion was reached by varying at will the deformation of the valence nucleons. Thus, the above conclusion does not necessarily hold in our case, where realistic calculations for more complex nuclei are performed. Actually, it was also found in Ref. [48] that the spin $M 1$ 
TABLE IV. Gap parameters for neutrons $\Delta_{n}$ and protons $\Delta_{p}$. Intrinsic quadrupole moments $Q_{0}$ and root-mean-square charge radii $\left\langle r^{2}\right\rangle^{1 / 2}$ calculated from the HF mean field with the force SG2. The quadrupole deformations $\beta_{\text {th }}$ are calculated from Eq. (12). The theoretical RPA spin $M 1$ strengths summed up to $12 \mathrm{MeV}$ and the corresponding energy-weighted sums are listed in the two last columns.

\begin{tabular}{lccccccc}
\hline \hline Nucleus & $\begin{array}{c}\Delta_{n} \\
{[\mathrm{MeV}]}\end{array}$ & $\begin{array}{c}\Delta_{p} \\
{[\mathrm{MeV}]}\end{array}$ & $\begin{array}{c}Q_{0}^{\text {th }} \\
{[e \mathrm{~b}]}\end{array}$ & $\begin{array}{c}\left\langle r^{2}\right\rangle_{\text {th }}^{1 / 2} \\
{[\mathrm{fm}]}\end{array}$ & $\beta_{\text {th }}$ & $\begin{array}{c}\sum_{\nu} B_{\sigma}(M 1, \nu) \\
{\left[\mu_{N}^{2}\right]}\end{array}$ & $\begin{array}{c}\sum_{\nu} E_{\nu} B_{\sigma}(M 1, \nu) \\
{\left[\mathrm{MeV} \mu_{N}^{2}\right]}\end{array}$ \\
\hline${ }^{142} \mathrm{Nd}$ & 1.4 & 1.2 & 0.041 & 4.902 & 0.002 & 6.5 & 53.2 \\
${ }^{146} \mathrm{Nd}$ & 0.9 & 1.2 & 2.764 & 4.967 & 0.148 & 8.2 & 64.9 \\
${ }^{148} \mathrm{Nd}$ & 1.1 & 1.4 & 3.673 & 5.007 & 0.194 & 8.5 & 68.3 \\
${ }^{150} \mathrm{Nd}$ & 1.2 & 1.3 & 4.884 & 5.053 & 0.253 & 9.8 & 77.4 \\
${ }^{144} \mathrm{Sm}$ & 1.1 & 1.1 & 0.043 & 4.921 & 0.002 & 7.2 & 56.8 \\
${ }^{148} \mathrm{Sm}$ & 1.0 & 1.4 & 2.667 & 4.987 & 0.137 & 7.8 & 62.7 \\
${ }^{150} \mathrm{Sm}$ & 1.2 & 1.6 & 3.899 & 5.030 & 0.197 & 8.3 & 68.2 \\
${ }^{152} \mathrm{Sm}$ & 1.2 & 1.3 & 5.507 & 5.081 & 0.273 & 10.0 & 78.8 \\
${ }^{154} \mathrm{Sm}$ & 1.1 & 1.2 & 6.599 & 5.133 & 0.320 & 11.1 & 85.8 \\
\hline \hline
\end{tabular}

strength remains finite at any deformation if more nucleons are added to the open shell.

Calculations using a Nilsson mean field and the quasiparticle Tamm-Dancoff approximation with Coriolis and separable spin and quadrupole residual interactions were performed in Ref. [23] for rare-earth nuclei ranging from ${ }^{140} \mathrm{Ce}$ to ${ }^{198} \mathrm{Pt}$. Results from QRPA calculations with a Woods-Saxon mean field and zero-range Landau-Migdal $[22]$ or separable $[13,14]$ residual interactions were reported for rare-earth nuclei from ${ }^{154} \mathrm{Sm}$ to ${ }^{184} \mathrm{~W}$ and for nuclei from ${ }^{150} \mathrm{Nd}$ to ${ }^{238} \mathrm{U}$, respectively. Although the systematics of the spin $M 1$ strength distribution was studied in that region, its deformation dependence was not discussed in said references. Common to these works is the appearance of a twohumped structure in the spin $M 1$ strength distributions. However, different approaches predict quite different strengths in the two bumps, as well as different overall strengths.

To study in detail the dependence on deformation, we focus the discussion on the two isotope chains that have a larger variation in the deformation parameter $\beta$, ${ }^{142,146,148,150} \mathrm{Nd}$ and ${ }^{144,148,150,152,154} \mathrm{Sm}$. For this detailed study we use pairing gaps chosen individually for each isotope instead of the uniform constant values used in the previous section. The adopted values, listed in Table IV, have been derived from the experimental odd-even mass differences. The corresponding new values of $Q_{0}$ and rms radii, obtained with these pairing gaps from our deformed selfconsistent calculations, are also listed in Table IV. These theoretical values can be compared with the experimental data from Table III.

The agreement of these global properties with experiment is similar to the previous case of constant gaps, presented in Table III. Three of the isotopes $\left({ }^{142} \mathrm{Nd},{ }^{144} \mathrm{Sm}\right.$, and $\left.{ }^{148} \mathrm{Sm}\right)$ considered in this section are not included in Table III. We quote here for completeness their experimental values: $Q_{0}^{\text {expt }}=2.690(60) e \mathrm{~b}$ [42] and $\left\langle r^{2}\right\rangle_{\text {expt }}^{1 / 2}=5.002(6) \mathrm{fm}$ [43] for ${ }^{148} \mathrm{Sm},\left\langle r^{2}\right\rangle_{\exp }^{1 / 2}=4.920 \mathrm{fm} \quad[43]$ for ${ }^{142} \mathrm{Nd}$, and $\left\langle r^{2}\right\rangle_{\text {expt }}^{1 / 2}=4.947(9) \mathrm{fm}[43]$ for ${ }^{144} \mathrm{Sm}$. The intrinsic quadrupole moments of the spherical nuclei ${ }^{142} \mathrm{Nd}$ and ${ }^{144} \mathrm{Sm}$ are zero. We do not quote the experimental values of $Q_{0}$ [42] for these two nuclei because the simple rotational model used in [42] to extract intrinsic quadrupole moments from the experimental $B(E 2)$ values is not meaningful for spherical nuclei, where $B(E 2)$ corresponds to a vibrational excitation and not to a static ground-state deformation. The quadrupole deformation parameters, listed in Table IV, are derived from the relationship

$$
\beta=\sqrt{\frac{\pi}{5}} \frac{Q_{0}^{p}}{Z\left\langle r_{p}^{2}\right\rangle},
$$

using the theoretical values of the quadrupole moments and rms radii. The last two columns in Table IV contain the calculated spin $M 1$ strengths summed up to $12 \mathrm{MeV}$, as well as the corresponding energy-weighted sums.

The available experimental information on $1^{+}$excitations in these chains of isotopes covers (i) the summed $M 1$ strength below $4 \mathrm{MeV}$ in each isotope [5,6] and (ii) the spin $M 1$ strength distributions of the most deformed nucleus in each chain, ${ }^{150} \mathrm{Nd}$ and ${ }^{154} \mathrm{Sm}[7,12]$. The experimental data (ii) are successfully described by our calculations. As already mentioned, it has been demonstrated experimentally that the summed $M 1$ strength below $4 \mathrm{MeV}$ is predominantly orbital and proportional to $\beta^{2}$. Hence, before discussing the deformation dependence of the spin $M 1$ strength, we would like to check that this property is also confirmed in the present theoretical framework.

\section{A. Low-lying excitations}

The theoretical spin (orbital) $M 1$ strength is obtained by putting the orbital (spin) gyromagnetic factors in the $M 1$ operator equal to zero. The total strength is obtained with the full $M 1$ operator containing both spin and orbital terms. We use the already mentioned effective spin gyromagnetic factors and bare orbital ones, $g_{\ell}^{p}=1, g_{\ell}^{n}=0$.

Our results obtained from the present model are compared in Fig. 10 to the available experimental data on summed low-lying $1^{+}$excitations. The orbital (solid dots) and total (stars) $M 1$ strengths summed up to $4 \mathrm{MeV}$ are plotted versus $\beta^{2}$ for the Sm and Nd isotopes. They are compared to the 


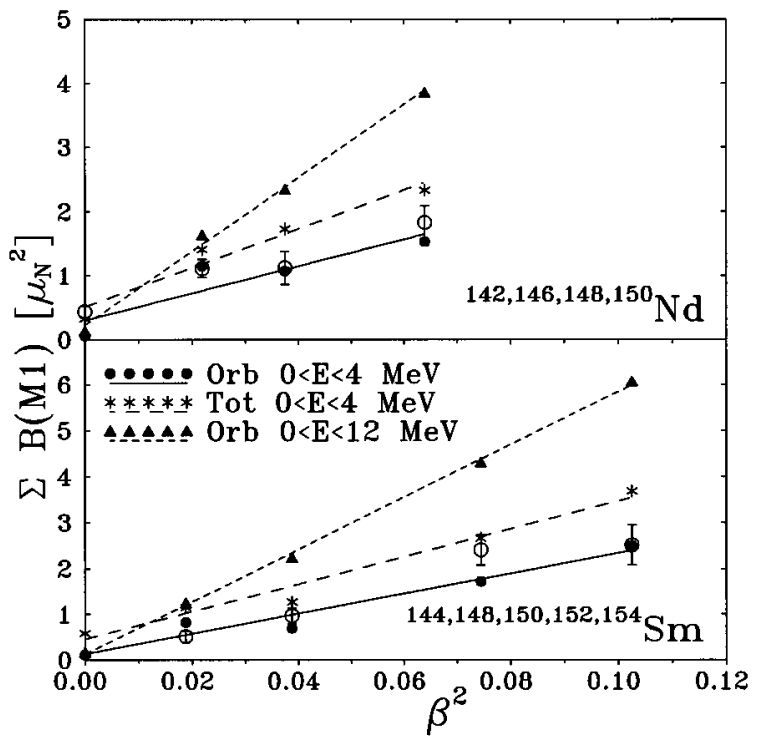

FIG. 10. Theoretical orbital (solid dots) and total (stars) $M 1$ strengths obtained with the SG2 force and summed up to $4 \mathrm{MeV}$ for the two chains of $\mathrm{Nd}$ and $\mathrm{Sm}$ isotopes versus the quadrupole deformation $\beta$, Eq. (12), squared. Triangles: theoretical orbital $M 1$ strengths summed up to $12 \mathrm{MeV}$. The lines are least-squares fits to the theoretical points. The experimental data for the total $M 1$ strength summed up to $4 \mathrm{MeV}$ (circles with error bars) are from Refs. [5,6] for $\mathrm{Nd}$ and $\mathrm{Sm}$, respectively.

experimental total $M 1$ strength below $4 \mathrm{MeV}[5,6]$ (circles with error bars). One can see from Fig. 10 that a $\beta^{2}$ dependence is exhibited to a good approximation by the theoretical orbital and total summed $M 1$ strengths. The agreement with experiment is at the same level as in different former approaches [14,25,27]; see Ref. [7] for a recent review.

Small deviations from the $\beta^{2}$ behavior take place in some isotopes. They are caused by the particular choice of the cutoff energy adopted for the summation of the strength. Small variations in the cutoff energy can have a local influence on the summed strength of some nuclei. A typical example of this situation on the experimental side is the case of ${ }^{142} \mathrm{Nd}$, which has an excitation at $4095 \mathrm{keV}$ with $B(M 1)=(0.42 \pm 0.08) \mu_{N}^{2}[6]$. This $B(M 1)$ value is, nevertheless, usually included in the reported $M 1$ strength below 4 $\mathrm{MeV}$. In order to avoid such fluctuations we also plot in Fig. 10 the theoretical orbital $M 1$ strength summed up to 12 $\mathrm{MeV}$, where such effects have been washed out, as seen from the reduced deviations of the triangles from the short-dashed straight lines.

Our calculated total $M 1$ strength summed up to $4 \mathrm{MeV}$ (stars in Fig. 10) does not go to zero in the spherical nuclei ${ }^{142} \mathrm{Nd}$ and ${ }^{144} \mathrm{Sm}$ as the orbital strength does. The nonvanishing low-lying strength at zero deformation originates obviously from spin $M 1$ excitations, which are also present in spherical nuclei. We will consider this strength in more detail below when discussing the spin $M 1$ strength distribution.

If one compares now the results in Fig. 10 with the corresponding results from Ref. [14] obtained with a WoodsSaxon potential, one can see that the discrepancy with experiment is reduced in some cases $\left({ }^{150} \mathrm{Nd}\right)$ but it still persists in other nuclei. The comparison reveals also that the difference between the orbital and the total $M 1$ strength is larger in the present case. This means that at low energy the spin admixtures are enhanced with respect to our previous calculations. The reason for this enhancement is twofold. On the one hand, the single-particle Hartree-Fock wave functions contain more spin mixing due to the spin-spin terms of the Skyrme interaction. On the other hand, the smaller coupling constant of the self-consistent spin-spin residual interaction $\left(K_{S}=88 \mathrm{MeV}\right.$ instead of $K_{S}=200 \mathrm{MeV}$ in Ref. [14]) moves less spin strength to higher energies.

To illustrate this effect, one can look at the ratio $\left(R_{O / S}\right)$ of the orbital to the spin $M 1$ transition matrix elements, as defined in Eq. (9) of Ref. [18]. The strongest $1^{+}$excitation in ${ }^{154} \mathrm{Sm}$ with $B(M 1)=0.9 \mu_{N}^{2}$ at $E=3.2 \mathrm{MeV}$ in our calculations has a ratio $R_{O / S}=3.5$, which is about 3 times smaller than the ratio obtained with a Woods-Saxon potential in Ref. [14]. The present value is also compatible with the experimental data [4] $\left(R_{O / S}>0.8\right)$.

It is worth pointing out that all the RPA $M 1$ transitions are included in the summed theoretical values shown in Fig. 10 but it would be also meaningful to sum only those contributions with $B(M 1)$ larger than the threshold below which the transitions cannot be distinguished experimentally from the background. The introduction of such a threshold in our calculations would reduce the theoretical strength. A reduction of the summed strength by typically $10 \%$ is found if one restricts the sums to excitations with $B(M 1)>0.1 \mu_{N}^{2}$

\section{B. Spin M1 strength distributions}

We proceed now to the study of the systematics of the spin $M 1$ strength distributions in the two chains of Sm and $\mathrm{Nd}$ isotopes. In order to understand better the general patterns of these distributions, it is advantageous to start first considering the unperturbed case where no spin-spin residual interaction is active yet $\left(H_{\mathrm{SS}}=0\right)$. This allows us to study separately the effect of the residual spin-spin interaction and the effect of deformation.

In Fig. 11 one can see the unperturbed $\left(H_{\mathrm{SS}}=0\right)$ spin $M 1$ strength distributions for $\mathrm{Nd}$ and $\mathrm{Sm}$. Observation of Fig. 11 reveals some interesting features when moving from almost spherical $\left({ }^{142} \mathrm{Nd},{ }^{144} \mathrm{Sm}\right)$ to strongly deformed $\left({ }^{150} \mathrm{Nd},{ }^{154} \mathrm{Sm}\right)$ nuclei. We discuss further only the results for $\mathrm{Nd}$ since the two chains of isotopes share common features and exhibit similar trends. The first typical feature is the existence of a small bump at about $3.5 \mathrm{MeV}$, which spreads in energy as the deformation increases. Most of the spin strength resides, however, at about $8 \mathrm{MeV}$ in the spherical limit. The strength of this peak decreases with increasing deformation. A new bump appears at $6 \mathrm{MeV}$ in the presence of deformation. It evolves with deformation in the opposite direction; i.e., its strength increases with the deformation. This peak carries a considerable amount of strength in the most deformed case of ${ }^{150} \mathrm{Nd}$.

The origin of this behavior has to be found in the microscopic structure of the individual excitations building the bumps. Looking at the structure of the QRPA wave functions one can learn that the bump at $3.5 \mathrm{MeV}$ originates mainly from the proton shell transition $2 d_{5 / 2}-2 d_{3 / 2}$ (when denoting particle-hole configurations, we specify the hole shell on the first position). As the deformation increases, further twoquasiparticle configurations become active and the transition 


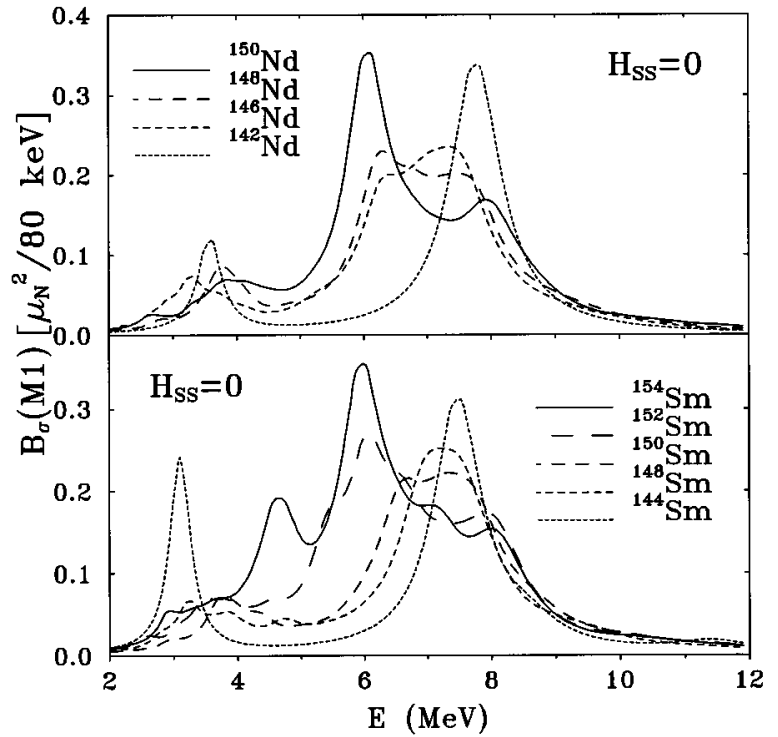

FIG. 11. Energy distributions of the theoretical unperturbed $\left(H_{\mathrm{SS}}=0\right)$ spin $M 1$ strength with $K^{\pi}=1^{+}$obtained with the SG2 force for the $\mathrm{Nd}$ and $\mathrm{Sm}$ isotopes. Units and Gaussian widths are as in Fig. 1.

becomes more fragmented. The peak at $6 \mathrm{MeV}$ originates mainly from the proton transition $1 g_{9 / 2}-1 g_{7 / 2}$. The fact that these excitations are not present in the spherical limit is easily understood since in this case both shells are below the Fermi level. Hence, it is not possible to build such a particlehole excitation, apart from a small mixing caused by the pairing smearing of the Fermi surface. As the deformation increases, the degeneracy of the spherical shells is removed and their components with different $K$ numbers spread in energy. Some of the $1 g_{7 / 2}$ components cross the Fermi level, enhancing quite dramatically the $M 1$ strength. When the deformation is large enough this proton $1 g_{9 / 2}-1 g_{7 / 2}$ dominant structure becomes more and more mixed with other configurations including neutrons.

The peak at $8 \mathrm{MeV}$ originates in the spherical case from the neutron $1 h_{11 / 2}-1 h_{9 / 2}$ transition. It is a single peak, although the folding procedure makes it wider. The strength in this peak becomes smaller and smaller with deformation. The reason for this behavior is similar to the mechanism producing the opposite effect in the peak at $6 \mathrm{MeV}$. In the spherical case the $1 h_{11 / 2}\left(1 h_{9 / 2}\right)$ shell is below (above) the Fermi level. On the other hand, the deformation and the increasing number of neutrons make the lower $K$ components of the $1 h_{9 / 2}$ shell move below the Fermi level in the deformed isotopes. The strength of the $1 h_{11 / 2}-1 h_{9 / 2}$ transition is therefore reduced. Again, when the deformation is large enough, a lot of extra proton and neutron configurations become active in this energy region and the leading $1 h_{11 / 2^{-}}$ $1 h_{9 / 2}$ transition becomes less important.

Once we understand the trends of the unperturbed strength distribution, it is easy to explain the more realistic results in Fig. 12, where the spin-spin interaction is included. The spin-spin interaction transfers strength (mainly isovector strength because $q=-0.95$ ) from the first peak at $6 \mathrm{MeV}$ to the second at $8 \mathrm{MeV}$. The whole strength is shifted also to higher energies, because this interaction is repulsive. The

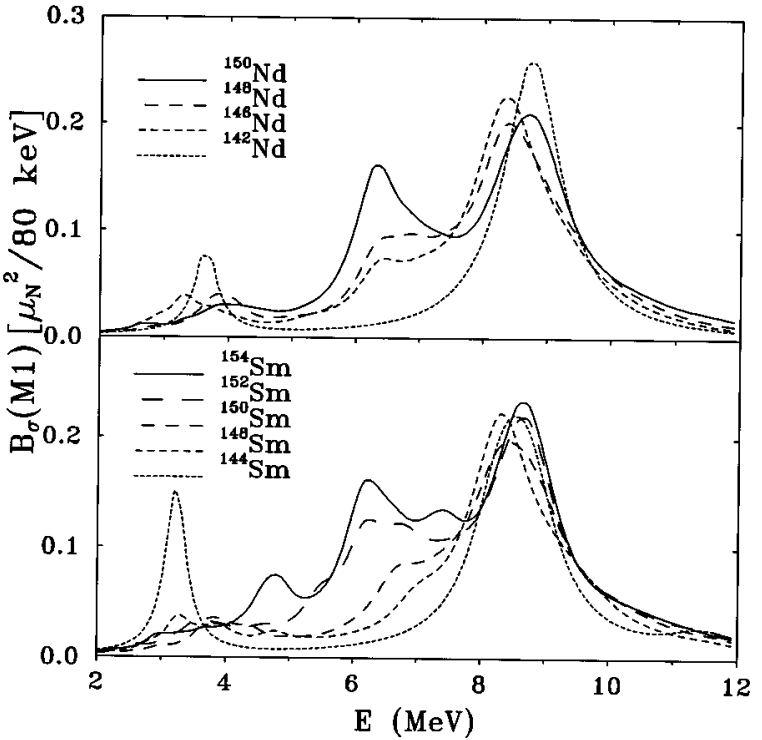

FIG. 12. Same as in Fig. 11 but with included residual spin-spin interaction $H_{\mathrm{SS}}$.

total spin $M 1$ strength is reduced due to the conservation of the energy-weighted sum rule in RPA.

Therefore, the small bump at $3.5 \mathrm{MeV}$ is slightly moved to higher energies and its strength is reduced. The strength contained in this peak in the spherical nuclei ${ }^{142} \mathrm{Nd}$ and ${ }^{144} \mathrm{Sm}$ is the total $M 1$ strength below $4 \mathrm{MeV}$ shown for them in Fig. 10. The $M 1$ strength found experimentally at 4095 $\mathrm{keV}$ in ${ }^{142} \mathrm{Nd}[6]$ corresponds in the present model to a spin excitation.

The situation in the peak at $6.5 \mathrm{MeV}$ is qualitatively similar. The behavior with respect to deformation found without spin-spin force (Fig. 11) is preserved now in Fig. 12 in the presence of this force, although the strength of the peak is reduced for all the isotopes. The original proton structure of this peak has almost disappeared because of deformation and because of the spin-spin interaction which makes it isoscalar.

Concerning the second peak at $8 \mathrm{MeV}$, we have two opposite effects which cancel more or less each other: first, the effect originating from the unperturbed case, where the increase in deformation results in a strength reduction. The second effect is the extra strength coming from the first peak, which is transferred by the isovector spin-spin force. Since more strength is transferred when the nucleus is more deformed, this effect compensates the former one with a net result of an almost steady peak at $8.5 \mathrm{MeV}$ independent of deformation. Again, this peak changes its original neutron character, inherent to the spherical unperturbed case, to a marked isovector character due to the deformation and the isovector spin-spin interaction (compare Figs. 2 and 3).

It should be mentioned that the excitations with $\Delta K=0$ and $|\Delta K|=1$ are degenerate in the spherical case. Therefore, the $\Delta K=0$ spin $M 1$ strength (not shown in Fig. 12) is $50 \%$ of the strength displayed for ${ }^{142} \mathrm{Nd}$ and ${ }^{144} \mathrm{Sm}$. Hence, the total spin $M 1$ strengths for ${ }^{142} \mathrm{Nd}$ and ${ }^{144} \mathrm{Sm}$ can be obtained from those depicted in Fig. 12 by multiplying with the scale factor 1.5. The contribution of these $\Delta K=0$ excitations is much smaller in the deformed case, as shown in Refs. $[21,22,41]$. Another remark to be made is that the spin 


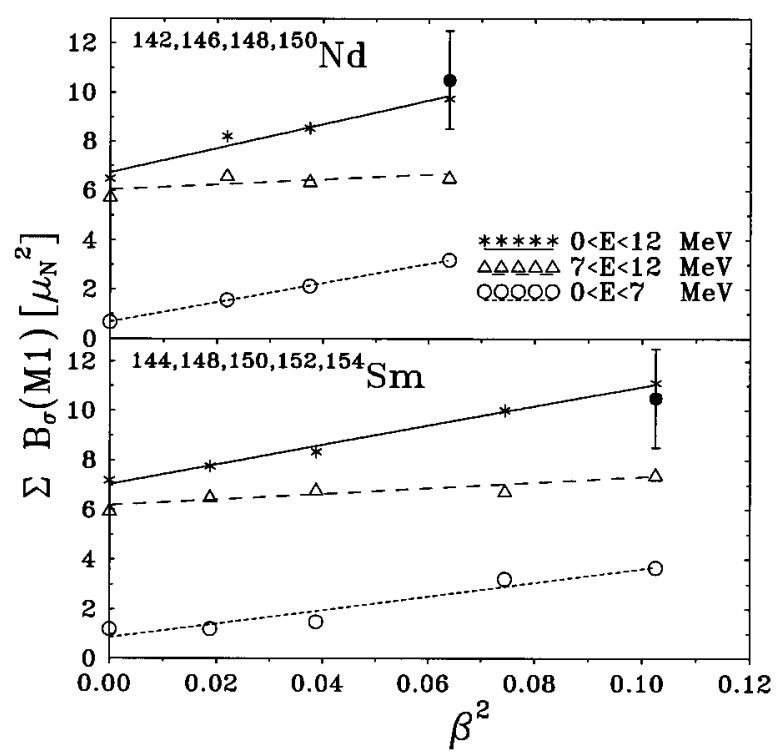

FIG. 13. Theoretical spin $M 1$ strength with $K^{\pi}=1^{+}$obtained with the SG2 force and summed up to $12 \mathrm{MeV}$ (stars) versus the quadrupole deformation $\beta$, Eq. (12), squared. Also shown is the theoretical spin $M 1$ strength contained in the energy ranges $0<E<7 \mathrm{MeV}$ (open circles) and $7<E<12 \mathrm{MeV}$ (triangles). The straight lines are least-squares fits to the theoretical points. The measured spin $M 1$ strengths in ${ }^{150} \mathrm{Nd}$ and ${ }^{154} \mathrm{Sm}$ [7] are displayed by dots with error bars.

$M 1$ strength with $\Delta K=0$ appears at about $10 \mathrm{MeV}$ when a separable spin-spin residual interaction is used in [41] on the example of ${ }^{154} \mathrm{Sm}$. Since our present calculations do not contain excitations with $\Delta K=0$, this strength could be an explanation for the small bump at around $10 \mathrm{MeV}$ seen experimentally. Work in this direction is in progress.

It is now worth looking in more detail at the dependence on the deformation of the $|\Delta K|=1$ spin strength. The spin $M 1$ strength contained within the energy range $0<E<12$ $\mathrm{MeV}$ (stars) is plotted in Fig. 13 for the two isotope chains. These strengths are plotted versus the deformation squared $\left(\beta^{2}\right)$ and the solid lines correspond to least-square fits to the theoretical points. We have plotted also the spin strengths summed in the intervals $0<E<7 \mathrm{MeV}$ (open circles) and $7<E<12 \mathrm{MeV}$ (triangles), which correspond roughly to the energy intervals of the first and second bumps in the spin $M 1$ strength distribution, respectively. The measured spin $M 1$ strengths for ${ }^{150} \mathrm{Nd}$ and ${ }^{154} \mathrm{Sm}$ are shown as dots with error bars.

One can see in Fig. 13 a clear $\beta^{2}$ dependence of the $|\Delta K|=1$ spin $M 1$ strength that originates mainly from the first peak below $7 \mathrm{MeV}$. Above this energy the strength remains almost independent of deformation, as seen from the almost horizontal long-dashed lines. It would be interesting to see whether this conclusion is modified when $\Delta K=0$ spin excitations are also included.

As a final remark, we would like to point out that the proportionality to $\beta^{2}$ of the summed spin strength with $|\Delta K|=1$ is consistent with the corresponding behavior of the summed orbital $M 1$ strength. To see this we notice that for any nonspurious $1_{\alpha}^{+}$excitation one has

$$
\left\langle 1_{\alpha}^{+}\left|J_{+}\right| 0\right\rangle=0,
$$

where $J$ is the total angular momentum operator. It follows that

$$
\left\langle 1_{\alpha}^{+}\left|S_{+}\right| 0\right\rangle=-\left\langle 1_{\alpha}^{+}\left|L_{+}\right| 0\right\rangle
$$

and

$$
\sum_{\alpha}\left|\left\langle 1_{\alpha}^{+}\left|S_{+}\right| 0\right\rangle\right|^{2}=\sum_{\alpha}\left|\left\langle 1_{\alpha}^{+}\left|L_{+}\right| 0\right\rangle\right|^{2} .
$$

The left-hand side in Eq. (15) is proportional to the summed isoscalar spin strength

$$
\sum_{\alpha} B_{\alpha}(M 1 ; \text { is }, s)=\frac{3}{16 \pi}\left(g_{s}^{n}+g_{s}^{p}\right)^{2} \sum_{\alpha}\left|\left\langle 1_{\alpha}^{+}\left|S_{+}\right| 0\right\rangle\right|^{2},
$$

which in turn is also proportional to the isovector spin strength. On the other hand the right-hand side in Eq. (15) is proportional to $\left\langle L_{\perp}^{2}\right\rangle$ (the mean value in the intrinsic ground state of the normal component of the orbital angular momentum operator squared [49]), which has been shown to be proportional to $\beta^{2}$ [25]. This implies that the total (isovector and isoscalar) spin $M 1$ strength should be proportional to $\beta^{2}$.

\section{SUMMARY AND CONCLUSIONS}

We have studied spin $M 1$ excitations in deformed nuclei within the QRPA formalism. What is new in the present approach is that we use a self-consistent mean field and residual interactions derived from the same two-body effective interaction of the Skyrme type. Once the Skyrme parametrization is chosen (by previous fits to nuclear matter and ground-state properties of spherical nuclei) and the gap constants of the pairing force are specified, there are no free parameters in the model Hamiltonian. The coupling strengths of the $H_{\mathrm{QQ}}$ and $H_{\mathrm{RV}}$ interactions are determined microscopically from the condition of rotational invariance. Those of the spin-spin residual interaction are derived from the parameters of the Skyrme force. Therefore, there is not a single parameter in the present approach fitted to the observables described here.

We compare the results on spin $M 1$ strength distributions obtained with different two-body density-dependent Skyrme interactions with and without inclusion of the spin-spin residual interaction $H_{\mathrm{SS}}$. We find that before including $H_{\mathrm{SS}}$ the profiles obtained with the various forces are qualitatively similar but the strength is displaced to higher energies for the Skyrme forces with larger spin-orbit interactions. When the $H_{\text {SS }}$ interaction is turned on the spin $M 1$ strength is shifted from low to higher energy for all the two-body densitydependent Skyrme forces considered here. The reason for this effect is that in all cases the isovector spin-spin residual interaction is repulsive since $G_{0}^{\prime}$ takes positive values between 0.3 and 0.5 . The isoscalar spin-spin residual interaction changes substantially for different Skyrme forces, but its effect on the spin $M 1$ strength distribution is negligible because of the Morpurgo factor. Once $H_{\mathrm{SS}}$ is included, the spin M1 strength distributions obtained with the various twobody density-dependent Skyrme forces do not differ much, but on the overall the force SG2 provides a better description 
of the available experimental data.

We compare the results on spin $M 1$ strength distributions obtained with the SG2 force with experimental data for the eight nuclei where the spin $M 1$ strength has been measured. There is a general agreement with the structure of the observed strength distribution for each nucleus. The twohumped structure in most nuclei is in agreement with experiment. The only feature missing in our results is the small bump in the $10 \mathrm{MeV}$ region, observed in ${ }^{150} \mathrm{Nd}$ and ${ }^{154} \mathrm{Sm}$, which can be regarded as preliminary data. The two-humped structure is already present in the mean-field calculations but the weak residual interaction $H_{\mathrm{SS}}$ improves the agreement with experiment in most cases.

Hence, we can conclude that the self-consistent formalism used here describes successfully the phenomenology of spin excitations in deformed nuclei and that the agreement found between theory and experiment constitutes a new piece of evidence in favor of the two-body density-dependent Skyrme-type effective interactions.

We present theoretical results on spin $M 1$ strength distributions of $I^{\pi} K=1^{+} 1$ excitations for 27 nuclei in various mass regions to study their common and distinguishing features.

We find that there are generally two peaks in the spin M1 strength distribution separated by $2-3 \mathrm{MeV}$, though in detail the structure of the profiles changes for each isotopic chain and the strength tends to be displaced to higher energies as the mass number decreases. We also observe that the strength of the first peak tends to decrease with decreasing deformation. This is in qualitative agreement with the experimental observations in ${ }^{208} \mathrm{~Pb}$ [9].
The dependence on deformation is studied in more detail in the two chains of isotopes ${ }^{142,146,148,150} \mathrm{Nd}$ and ${ }_{144,148,150,152,154} \mathrm{Sm}$. We have first checked that the present theoretical approach provides a very reasonable description of the $\beta^{2}$ dependence of the summed low-lying (predominantly orbital) $M 1$ strength. Then, we have analyzed the dependence on deformation of the spin $M 1$ strength. We find a quadratic dependence on the deformation parameter $\beta$ of the spin $M 1$ strength summed up to $12 \mathrm{MeV}$. This energy range includes practically the whole spin $M 1$ strength obtained in our microscopic QRPA calculations. The $\beta^{2}$ dependence arises mainly from the strength contained in the first bump of the spin $M 1$ strength distribution. While the second bump remains rather stable with deformation, the first bump decreases with decreasing deformation. These features are the net result from the interplay between the deformation and the spin-spin residual interaction acting in opposite directions.

It would be very interesting to extend the $\left(p, p^{\prime}\right)$ experiments to other regions, and particularly to less deformed isotopes in order to obtain a more complete systematics of the spin $M 1$ excitations. In particular, it would be interesting to test experimentally the new theoretical predictions presented here.

\section{ACKNOWLEDGMENTS}

Thanks are due to A. Faessler, D. Frekers, A. Richter, and H.J. Wörtche for encouraging discussions. The work is supported by the EC program "Human Capital and Mobility" under Contracts No. CHRX-CT 93-0323 and CHRX-CT 940562. P.S. and E.M.G. are indebted to DGICYT (Spain) for partial financial support under Contract No. 92/0021-C02-01.
[1] D. Bohle, A. Richter, W. Steffen, A.E.L. Dieperink, N. Lo Iudice, F. Palumbo, and O. Scholten, Phys. Lett. 137B, 27 (1984).

[2] A. Richter, Nucl. Phys. A522, 139c (1991); A553, 417c (1993).

[3] U. Kneissl, Prog. Part. Nucl. Phys. 24, 41 (1990).

[4] C. Djalali, N. Marty, M. Morlet, A. Willis, J.C. Jourdain, D. Bohle, U. Hartmann, G. Küchler, A. Richter, G. Caskey, G.M. Crawley, and A. Galonsky, Phys. Lett. B 164, 269 (1985).

[5] W. Ziegler, C. Rangacharyulu, A. Richter, and C. Spieler, Phys. Rev. Lett. 65, 2515 (1990); W. Ziegler, N. Huxel, P. von Neumann-Cosel, C. Rangacharyulu, A. Richter, C. Spieler, C. de Coster, and K. Heyde, Nucl. Phys. A564, 366 (1993).

[6] J. Margraf, R.D. Heil, U. Kneissl, U. Maier, H.H. Pitz, H. Friedrichs, S. Linderstruth, B. Schlitt, C. Wesselborg, P. von Brentano, R.D. Herzberg, and A. Zilges, Phys. Rev. C 47, 1474 (1993).

[7] A. Richter, Prog. Part. Nucl. Phys. 34, 261 (1995).

[8] D. Frekers, H.J. Wörtche, A. Richter, R. Abegg, R.E. Azuma, A. Celler, C. Chan, T.E. Drake, R. Helmer, K.P. Jackson, J.D. King, C.A. Miller, R. Schubank, M.C. Vetterli, and S. Yen, Phys. Lett. B 244, 178 (1990).

[9] R.M. Laszewski, R. Alarcon, D.S. Dale, and S.D. Hoblit, Phys. Rev. Lett. 61, 1710 (1988).

[10] D. Cha, B. Schwesinger, J. Wambach, and J. Speth, Nucl. Phys. A430, 313 (1988).
[11] D. Frekers and H.J. Wörtche, TRIUMF Annual Report, Vancouver, Canada, 1991, p. 45; and (private communication).

[12] H.J. Wörtche, Ph.D. thesis, Technischen Hochschule Darmstadt, Germany, 1994.

[13] P. Sarriguren, E. Moya de Guerra, R. Nojarov, and A. Faessler, J. Phys. G 19, 291 (1993).

[14] P. Sarriguren, E. Moya de Guerra, R. Nojarov, and A. Faessler, J. Phys. G 20, 315 (1994).

[15] D. Vautherin and D.M. Brink, Phys. Rev. C 5, 626 (1972); D. Vautherin, ibid. 7, 296 (1973).

[16] G.F. Bertsch and S.F. Tsai, Phys. Rep. 18, 127 (1975).

[17] N. Van Giai and H. Sagawa, Phys. Lett. 106B, 379 (1981).

[18] R. Nojarov and A. Faessler, Nucl. Phys. A484, 1 (1988).

[19] A. Faessler and R. Nojarov, Phys. Rev. C 41, 1243 (1990).

[20] R. Nojarov, A. Faessler, P. Sarriguren, E. Moya de Guerra, and M. Grigorescu, Nucl. Phys. A563, 349 (1993).

[21] D. Zawischa, M. Macfarlane, and J. Speth, Phys. Rev. C 42, 1461 (1990).

[22] D. Zawischa and J. Speth, Phys. Lett. B 252, 4 (1990).

[23] C. de Coster, K. Heyde, and A. Richter, Nucl. Phys. A542, 375 (1992).

[24] C. Magnusson, Phys. Scr. 43, 460 (1991).

[25] E. Garrido, E. Moya de Guerra, P. Sarriguren, and J.M. Udías, Phys. Rev. C 44, R1250 (1991).

[26] L. Zamick and D.C. Zheng, Phys. Rev. C 44, 2522 (1991); 46, 
2106 (1992); E. Moya de Guerra and L. Zamick, ibid. 47, 2604 (1993).

[27] J.N. Ginocchio, Phys. Lett. B 265, 6 (1991); I. Hamamoto and C. Magnusson, ibid. 260, 6 (1991); K. Heyde and C. De Coster, Phys. Rev. C 44, R2262 (1991); K. Heyde K, C. De Coster, A. Richter, and H.J. Wörtche, Nucl. Phys. A549, 103 (1992); N. Lo Iudice and A. Richter, Phys. Lett. B 304, 193 (1993).

[28] H. Flocard, P. Quentin, and D. Vautherin, Phys. Lett. 46B, 304 (1973); P. Quentin and H. Flocard, Annu. Rev. Nucl. Part. Sci. 28, 253 (1978); P. Bonche, H. Flocard, P.H. Heenen, S.J. Krieger, and M.S. Weiss, Nucl. Phys. A443, 39 (1985).

[29] M. Vallieres and D.W.L. Sprung, Can. J. Phys. 56, 1190 (1978).

[30] M. Waroquier, K. Heyde, and G. Wenes, Nucl. Phys. A404, 269 (1983); M. Waroquier, J. Ryckebusch, J. Moreau, K. Heyde, N. Blasi, S.Y. Werf, and G. Wenes, Phys. Rep. 148, 249 (1987).

[31] M. Beiner, H. Flocard, N. Van Giai, and P. Quentin, Nucl. Phys. A238, 29 (1975).

[32] S.O. Bäckmann, A.D. Jackson, and J. Speth, Phys. Lett. B 56, 209 (1975).

[33] H.S. Köhler, Nucl. Phys. A258, 301 (1976).

[34] S. Krewald, V. Klemt, J. Speth, and A. Faessler, Nucl. Phys. A281, 166 (1977).
[35] J. Bartel, P. Quentin, M. Brack, C. Guet, and H.B. Hakansson, Nucl. Phys. A386, 79 (1982).

[36] J. Friedrich and P.G. Reinhard, Phys. Rev. C 33, 335 (1986).

[37] K.F. Liu, H.Luo, Z. Ma, and Q. Shen, Nucl. Phys. A534, 1 (1991).

[38] B.D. Chang, Phys. Lett. B 56, 205 (1975).

[39] M.I. Baznat and N.I. Pyatov, Sov. J. Nucl. Phys. 21, 365 (1975).

[40] A.B. Migdal, Theory of Finite Fermi Systems (Interscience, New York, 1967).

[41] D. Zawischa and J. Speth, Nucl. Phys. A569, 343c (1994).

[42] S. Raman, C.H. Malarkey, W.T. Milner, C.W. Nestor, Jr., and P.H. Stelson, At. Data Nucl. Data Tables 36, 1 (1987).

[43] H. de Vries, C.W. de Jager, and C. de Vries, At. Data Nucl. Data Tables 36, 495 (1987).

[44] Y. Tanaka, Y. Oda, F. Petrovich, and R.K. Sheline, Phys. Lett. 83B, 279 (1979).

[45] C. de Coster and K. Heyde, Phys. Rev. Lett. 66, 2456 (1991).

[46] K. Heyde, C. de Coster, S. Rombouts, and S.J. Freeman, Nucl. Phys. A596, (1996) 30.

[47] G. Morpurgo, Phys. Rev. 110, 72 (1958).

[48] L. Zamick, D.C. Zheng, and E. Moya de Guerra, Phys. Rev. C 39, 2370 (1989); N. Auerbach, D.C. Zheng, L. Zamick, and B.A. Brown, Phys. Lett. B 304, 17 (1993).

[49] E. Moya de Guerra, Phys. Rep. 138, 409 (1987). 